\title{
Analysis of Alternative Massive MIMO Designs Superimposed Pilots and Mixed-ADCs
}

Daniel Verenzuela

\section{EOMMUNICATION Ş̄stems}

Division of Communication Systems Department of Electrical Engineering (ISY) Linköping University, 58183 Linköping, Sweden www.commsys.isy.liu.se

Linköping 2018 
This is a Swedish Licentiate Thesis.

The Licentiate degree comprises 120 ECTS credits of postgraduate studies.

\section{Analysis of Alternative Massive MIMO Designs}

(C) 2018 Daniel Verenzuela, unless otherwise noted.

ISBN 978-91-7685-323-8

ISSN 0280-7971

Printed in Sweden by LiU-Tryck, Linköping 2018 


\section{Abstract}

The development of information and communication technologies (ICT) provides the means for reaching global connectivity that can help humanity progress and prosper. This comes with high demands on data traffic and number of connected devices which are rapidly growing and need to be met by technological development. Massive MIMO, where MIMO stands for multiple-input multiple-output, is envisioned as a fundamental component of next generation wireless communications for its ability to provide high spectral and energy efficiency, SE and EE, respectively. The key feature of this technology is the use of a large number of antennas at the base stations (BS) to spatially multiplex several user equipments (UEs).

In the development of new technologies like Massive MIMO, many design alternatives need to be evaluated and compared in order to find the best operating point with a preferable tradeoff between high performance and low cost. In this thesis, two alternative designs for signal processing and hardware in Massive MIMO are studied and compared with the baseline operation in terms of SE, EE, and power consumption. The first design is called superimposed pilot (SP) transmission and is based on superimposing pilot and data symbols to remove the overhead from pilot transmission and reduce pilot contamination. The second design is mixed analog-to-digital converters (ADCs) and it aims at balancing high performance and low complexity by allowing different ADC bit resolutions across the BS antennas.

The results show that the baseline operation of Massive MIMO, properly optimized, is the preferred choice. However, SP and mixed ADCs still have room for improvement and further study is needed to ascertain the full capabilities of these alternative designs. 


\section{Resumen}

El desarrollo en tecnologías de información y comunicación (en inglés, ICT) provee los medios para alcanzar la conectividad global que puede ayudar a la humanidad a progresar y prosperar. Esto implica que el avance tecnológico debe satisfacer la alta demanda de tráfico de data y número de equipos conectados que se encuentra en rápido crecimiento. La tecnología de múltipleentrada múltiple-salida masiva, en inglés Massive MIMO, se considera una pieza fundamental de la nueva generación de comunicaciones inalámbricas debido a su capacidad de proveer una alta eficiencia espectral y energética (en inglés, SE y EE, respectivamente). Esta tecnología está caracterizada fundamentalmente por el uso de un alto número de antenas en la estación base (en inglés, BS) para multiplexar a varios usuarios en el espacio.

En el desarrollo de nuevas tecnologías como Massive MIMO, muchas alternativas de diseño necesitan ser evaluadas y comparadas para encontrar el mejor punto de operación con un balance conveniente entre alto desempeño y bajo costo. En esta tesis, dos alternativas de diseño para el procesamiento de señales y el hardware de Massive MIMO son estudiadas y comparadas con la operación del diseño base en términos de SE, EE y consumo de potencia. El primer diseño se denomina transmisión de pilotos superpuestos (en inglés, SP) y está basado en la superposición de símbolos pilotos y de datos para eliminar la sobrecarga debido a la transmisión de señales piloto y reducir la contaminación que estas producen (en inglés pilot contamination). El secundo diseño se denomina conversores analógico-a-digital (en inglés, $\mathrm{ADC}$ ) mixtos (en inglés, mixed ADCs) y se basa en permitir distintas resoluciones de bit en los ADC de las antenas en la estación base.

Los resultados muestran que el diseño base de Massive MIMO, cuando esta optimizado de manera apropiada, es la opción preferida. Sin embargo, SP y mixed ADCs todavía tienen potencial para mejorar su desempeño y se requiere más investigación para evaluar todos los beneficios que estos diseños pueden ofrecer. 


\section{Acknowledgments}

I have always been inclined to pursue knowledge and understanding in all aspects of life. In my path through academic research I have realized the importance of having a nurturing environment where ideas can emerge and develop. To successfully navigate in the deep waters of academic research, having the right guidance is of paramount importance. It is an honour for me to have Dr. Emil Björnson as my academic supervisor and mentor, without whom, this work would not have been possible. His expert advice and mentoring extends from the most general matters like finding research directions and explaining complicated theories, to the finest details in long mathematical expressions and finding that ever elusive typo. His vast knowledge and expertise in many different areas, his commitment towards his students, his dedication and passion towards excellence, have been a beacon that has guided and inspired me to be the researcher that I am today, I am truly grateful for his tutoring.

During the past couple of years, it has been a privilege to work in a group that has the highest standards for academic research. Therefore, I would like to thank Dr. Erik G. Larsson for giving me the opportunity to join his research group. His expert advice, teaching and commitment to high quality research have been fundamental for my development as a researcher.

Obtaining expert advise and feedback from diverse sources is essential to become a researcher. I would like to thank my co-supervisors Dr. Luca Sanguinetti (University of Pisa) and Dr. Michail Matthaiou (Queen's University Belfast). I thank Dr. Luca Sanguinetti for providing expert feedback, helping me navigate through difficult reviews, and encouraging further collaborations. I thank Dr. Michail Matthaiou for sharing his expert knowledge on complicated hardware topics, and giving advice and feedback on my research. Their dedication and support have help me achieve the results that are found in this thesis.

The never ending discussions and debates on research directions, complicated homework problems, basic theory concepts, and new publications, among others, have all been part of my education in research, and I thank 
my colleagues from the division of communication systems from 2015 and onwards for sponsoring this essential component of my education.

Family is, and has always been, at the core of my motivation to pursue excellence. They provide the never ending support and encouragement for me to be the best I can be in all aspects of life. I dedicate this thesis to: my parents, Juan y Coro; my brothers, Juan y David; my big family, "los Verenzuela-Moreno"; and last but not least my loving wife, Jeannine, who inspires me to be better every day.

Daniel Verenzuela Linköping, March 2018 


\section{Notation}

This is a summary of the basic notation used in the introduction of this thesis. Note also that additional terms are presented and defined along the text. Matrices are denoted by upper-case boldface letters (e.g., X) and column vectors are denoted by lower-case bold face letters (e.g., $\mathrm{x}$ ).

The following notation is used in this thesis:

$\mathbb{C} \quad$ Set of complex numbers

$\mathcal{C N}\left(0, \sigma^{2}\right) \quad$ Circularly symmetric complex Gaussian distribution with variance $\sigma^{2}$

$\operatorname{det}(\cdot) \quad$ Determinant operator

$\mathbb{E}\{\cdot\} \quad$ Expected value operator

$\mathbf{I}_{M} \quad$ Identity matrix of size $M \times M$

$\log _{2}(\cdot) \quad$ Logarithm function with base 2

$\mathbb{R} \quad$ Set of real numbers

$\operatorname{tr}(\cdot) \quad$ Trace operator

$\operatorname{Var}\{\cdot\} \quad$ Variance operator

$\mathbb{Z}_{+} \quad$ Set of positive integer numbers

$[\mathbf{X}]_{i j} \quad$ Element in row $i$ and column $j$ of matrix $\mathbf{X}$

$[\mathbf{x}]_{j} \quad$ Element $j$ of column vector $\mathbf{x}$

$|\cdot| \quad$ Absolute value operator

$\|\cdot\| \quad$ Euclidean norm operator

$(\cdot)^{*} \quad$ Conjugate operator

$(\cdot)^{T} \quad$ Transpose operator

$(\cdot)^{H} \quad$ Conjugate transpose operator 


\section{Abbreviations}

$\begin{array}{ll}\text { ADC } & \text { Analog-to-digital Converter } \\ \text { BER } & \text { Bit Error Rate } \\ \text { BS } & \text { Base Station } \\ \text { CSI } & \text { Channel State Information } \\ \text { DL } & \text { Downlink } \\ \text { EE } & \text { Energy Efficiency } \\ \text { FoM } & \text { Figure-of-Merit } \\ \text { ICT } & \text { Information and Communication Technologies } \\ \text { i.i.d. } & \text { independent identically distributed } \\ \text { LMMSE } & \text { Linear Minimum Mean Squared Error } \\ \text { MIMO } & \text { Multiple-Input Multiple-Output } \\ \text { mMMSE } & \text { multicell Minimum Mean Squared Error } \\ \text { MMSE } & \text { Minimum Mean Squared Error } \\ \text { MRC } & \text { Maximum Ratio Combining } \\ \text { MRT } & \text { Maximum Ratio Transmission } \\ \text { MSE } & \text { Mean Squared Error } \\ \text { MU-MIMO } & \text { Multiuser Multiple-Input Multiple-Output } \\ \text { PPP } & \text { Poisson Point Process } \\ \text { RF } & \text { Radio Frequency } \\ \text { RP } & \text { Regular Pilot transmission } \\ \text { SE } & \text { Spectral Efficiency } \\ \text { SER } & \text { Symbol Error Rate } \\ \text { SIMO } & \text { Single-Input Multiple-Output } \\ \text { SINR } & \text { Signal-to-Interference-plus-Noise Ratio } \\ \text { SISO } & \text { Single-Input Single-Output } \\ \text { SNR } & \text { Signal-to-Noise Ratio } \\ \text { SP } & \text { Superimposed Pilot transmission } \\ \text { TDD } & \text { Time Division Duplexing } \\ \text { UE } & \text { User Equipment } \\ \text { UL } & \text { Uplink } \\ \text { ZF } & \text { Zero forcing } \\ & \end{array}$




\section{Contents}

$\begin{array}{ll}\text { Introduction } & \mathbf{1}\end{array}$

1 Motivation $\quad 3$

1.1 Superimposed Pilots . . . . . . . . . . . . . 5

1.2 Hardware Design . . . . . . . . . . . . . . . 7

1.3 Contributions of this Thesis . . . . . . . . . . . 8

1.4 Included Papers . . . . . . . . . . . . . . . . 9

1.5 Excluded Papers $\ldots \ldots \ldots \ldots$. . . . . . . . . . . 11

2 Massive MIMO 13

2.1 Communication Channel . . . . . . . . . . . . . . 13

2.2 Properties of Massive MIMO Channels . . . . . . . . . . . 17

2.3 Channel Estimation . . . . . . . . . . . . . . 20

2.4 Capacity Analysis . . . . . . . . . . . . . . . . . . 23

2.5 Performance of Massive MIMO . . . . . . . . . . . . 26

3 Alternative Massive MIMO Designs 33

3.1 Superimposed Pilots . . . . . . . . . . . . . . 33

3.2 Hardware Impairments Optimization $\ldots \ldots \ldots \ldots$

4 Summary, Conclusions and Future Work 55

$\begin{array}{ll}\text { Bibliography } & 57\end{array}$

Included Papers $\quad 63$

A Spectral and Energy Efficiency of Superimposed Pilots in Uplink Massive MIMO

1 Introduction . . . . . . . . . . . . . . . 67

2 Network Model . . . . . . . . . . . . . . . . 70

3 Channel Estimation . . . . . . . . . . . . . 72

3.1 Regular Pilots . . . . . . . . . . . . . 72 
$3.2 \quad$ Superimposed Pilots . . . . . . . . . . . . . . . . 74

4 Achievable Rates with MRC . . . . . . . . . . . . . . 75

4.1 Random Pilot Allocation . . . . . . . . . . . 76

4.2 Regular Pilots . . . . . . . . . . . . . . . 77

4.3 Superimposed Pilots . . . . . . . . . . . . . 78

5 Analysis of Achievable Rates . . . . . . . . . . . . . . 84

6 Performance Comparison under Random Deployment . . . . 86

6.1 Energy Efficiency Modeling . . . . . . . . . . . . . 89

7 Numerical Results . . . . . . . . . . . . . . . . . . 90

8 Conclusions . . . . . . . . . . . . . . . . . 94

References ...................... 101

B Joint UL and DL Spectral Efficiency Optimization of Superimposed Pilots in Massive MIMO 105

1 Introduction . . . . . . . . . . . . . . . . . . 107

2 System Model . . . . . . . . . . . . . . . . . . . . 109

3 Channel Estimation . . . . . . . . . . . . . . . . 110

4 DL Achievable Rates . . . . . . . . . . . . . . . . . . . . . . 112

5 SE Comparison between RPs and SPs . . . . . . . . . . . 115

5.1 Multiobjective Optimization with RPs and SPs . . . 116

6 Numerical Results . . . . . . . . . . . . . . . . . . . 118

7 Conclusion ...................... 121

References ...................... 122

C Hardware Design and Optimal ADC Resolution for Up$\begin{array}{lr}\text { link Massive MIMO Systems } & 127\end{array}$

1 Introduction . . . . . . . . . . . . . . . . . . . . . . 129

2 System Model . . . . . . . . . . . . . . . . . . . . 130

3 Achievable Rate with Imperfect CSI . . . . . . . . . . . . . . 132

3.1 Power Consumption Model . . . . . . . . . . . . . . 134

4 Optimal Hardware Impairments Trade-off . . . . . . . . . . 136

5 Numerical Results . . . . . . . . . . . . . . . . . . . . 137

6 Conclusion ..................... 138

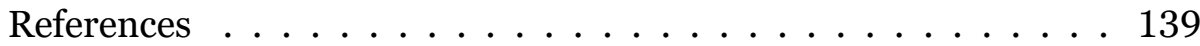

D Per-Antenna Hardware Optimization and Mixed Resolution ADCs in Uplink Massive MIMO 143

1 Introduction . . . . . . . . . . . . . . . . . 145

2 System Model . . . . . . . . . . . . . . . . . . . . . . 146

3 Channel Estimation . . . . . . . . . . . . . . . . . . 147

4 Achievable SE . . . . . . . . . . . . . . . . 149 
5 Effect of Mixed ADC Resolution _. . . . . . . . . . . . 150

$5.1 \quad$ Impact on Achievable SE . . . . . . . . . . . 150

5.2 Impact on Power Consumption . . . . . . . . . . . . 153

6 Numerical Results . . . . . . . . . . . . . . . . 153

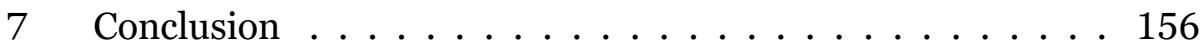

References ....................... 156 

Introduction 



\section{Chapter 1}

\section{Motivation}

Since the beginning of the 21st century there has been a massive expansion of information and communication technologies (ICT) all over the world. The Internet has become an essential part of our lives in developed societies and it has brought information and tools for progress to developing countries. In particular, wireless communications allow access to information and connectivity in highly mobile situations as well as in remote locations. The possibility to access information and have means of communication in a global scale brings us closer together and may help humanity to further develop and prosper.

To achieve global connectivity, mobile communication systems need to accommodate a massive amount of data traffic and number of connected devices while having low energy consumption. The mobile data traffic is expected to increase at an exponential rate for the foreseeable future and by the early 2020's there will be 1.5 mobile devices per person in the world, most of which will be smartphones demanding high data rates $[1,2]$. To meet the growing demand, the throughput of mobile networks needs to be increased by orders of magnitude [3]. On the other hand, the energy consumption and carbon footprint of the ICT industry is becoming an important societal and environmental concern $[4,5]$. This means that the throughput needs to increase without incrementing the current energy consumption of mobile networks, which calls for orders of magnitude improvements in energy efficiency (EE).

In cellular networks, the total throughput is defined as

$$
\mathrm{R}[\mathrm{bit} / \mathrm{s}]=\underbrace{N_{\text {cell }}}_{\text {Number of cells }} \times \underbrace{B_{\mathrm{W}}[\mathrm{Hz}]}_{\text {Bandwidth }} \times \underbrace{\mathrm{SE}[\mathrm{bit} / \mathrm{s} / \mathrm{Hz} / \text { cell }]}_{\text {Spectral efficiency }}
$$

and to improve it; either more cells, more bandwidth, or higher spectral 
efficiency (SE) is needed. Adding more cells, known as cell densification, has been the main source of throughput improvement over the past decades. However, as cells are placed closer together interference increases up to the point in which the throughput saturates and starts to decrease [6]. On the other hand, the bandwidth is limited by the amount of radio frequency spectrum that is available. Increasing the SE is a viable way of improving the throughput, however it comes at the cost of adding more complexity into the network which may result in higher power consumption. This thesis focuses on increasing the SE while considering its impact on the power consumption. The total EE of a cellular network is defined as

$$
\mathrm{EE}[\mathrm{bit} / \mathrm{Joule}]=\frac{N_{\text {cell }} \times B_{\mathrm{W}}[\mathrm{Hz}] \times \mathrm{SE}[\mathrm{bit} / \mathrm{s} / \mathrm{Hz} / \mathrm{cell}]}{\text { Power consumption }[\mathrm{Joule} / \mathrm{s}]},
$$

which means that reducing the power consumption and improving the SE results in a EE increase. In the development of the next generation wireless communication systems, the Massive MIMO technology, where MIMO stands for multiple-input multiple-output, has been proposed for its ability to provide both high SE and EE $[7,8]$. In this thesis, Massive MIMO is defined as a cellular multiuser MIMO (MU-MIMO) technology where the base stations (BSs) are equipped with a large number of antennas (e.g., hundreds or thousands) to simultaneously communicate with several (e.g., tens or hundreds) user equipments (UEs) in the same time-frequency resources. The main advantages of Massive MIMO are (see Chapter 2 for a more detailed explanation):

\section{Spatial multiplexing.}

The BSs separate the signals received from (and transmitted to) the UEs in the spatial domain such that multiple parallel data streams can be supported at the same time and in the same frequency band. This results in a linear increase of the SE per cell proportional to the number of served UEs.

\section{Coherent processing gain.}

The BSs process the signals received from (and transmitted to) each antenna element coherently. Here, coherent signal processing, means that the phase and amplitude of signals received from (or transmitted to) each antenna element are processed so that they add constructively when they are received from (or at) desired points in space where the UEs are located. This translates into a logarithmic gain in the SE per UE with respect to the number of BS antennas. Due to the large number of BS antennas, high SE per UE can be supported. 


\section{Transmission power reduction.}

Thanks to the coherent processing gain, the transmission power can be scaled down proportionally to the square root of the number of BS antennas while still increasing the SE.

\section{Linear processing.}

The large number of BS antennas induces two properties in the channels between the BS and the UEs called asymptotic favorable propagation and channel hardening. These properties allow the use of linear processing techniques that simplify the implementation while providing high SE.

\section{Low end hardware.}

Since there are many BS antennas, the coherent processing allows the hardware quality of each antenna branch to be relaxed, reducing the power consumption while supporting high SE, and in turn, high EE.

In the development and implementation of any new technology, there are always challenges that need to be surpassed and Massive MIMO is no exception. This leads to many different design proposals that need to be assessed and compared in a rigorous and fair manner to find the best possible system design. While [7] describes the baseline Massive MIMO system design, this thesis analyzes alternative designs that have been proposed to overcome some of the challenges of this technology.

\subsection{Superimposed Pilots}

In Massive MIMO, performing coherent signal processing is essential. This can be achieved by obtaining channel state information (CSI) from pilot transmission which introduces an overhead of transmitted pilot symbols; or by performing blind channel estimation and data detection. The latter is an interesting field of study, however these methods often have high complexity and require both large time-frequency resources and number of BS antennas to perform well [9-11]. Thus, this thesis is focused on the former, that is, estimating the channels between the BS and served UEs based on the transmission of known pilot sequences. These pilots allow the BS to identify and separate the channels from different UEs and perform channel estimation. In this thesis, a time division duplexing (TDD) protocol is assumed in which pilot sequences are transmitted by the UEs in the uplink (UL) from which the BS estimates the channels to the UEs. These channel estimates are then used to process the signals in the UL and downlink (DL) by virtue of channel reciprocity $[7,8]$. 
The communication channel is continuously changing both in time and frequency. Thus, the estimation and communication procedures need to happen in a short time and frequency block where the channel can be considered to be time invariant and frequency flat. This time-frequency block is called the coherence block and it contains a limited number of information symbols (see Chapter 2 for more details). This means that the number of available pilot sequences is limited, and in a multicell system, pilots need to be reused among cells. When UEs share the same pilot sequence, the channel estimates obtained at the BS are contaminated by interference which diminishes the estimation quality and reduces the SE $[7,8]$. This effect is called pilot contamination, and in uncorrelated channels, it leads to interfering terms that scale proportionally to the number of BS antennas.

There is a wide literature on mitigating the pilot contamination effect, as an example [7, 8, 12-18]. One approach, is to use the spatial correlation between the signals received at different BS antenna elements to separate UEs with the same pilot, as done in [8,12-14]. Another approach, is to increase the pilot overhead and allow for longer pilot sequences [7,15-18]. In a multicell system, the longer the pilot sequences, the less often they need to be reused in the network, thus the pilot contamination effect is reduced by the increased pathloss between UEs sharing the same pilot. However, having a larger pilot overhead reduces the number of symbols available for data transmission. The tradeoff between reducing the pilot contamination and having low pilot overhead is studied in [17] concluding that a pilot overhead between $5 \%$ and $40 \%$ maximizes the sum throughput.

In the aforementioned works, the transmission of pilot and data symbols is done separately within the coherence block to reduce interference in the channel estimation process. This method is known in the literature as regular pilot (RP) transmission. An alternative method is to simultaneously transmit a superposition of pilot and data symbols, which is referred to as superimposed pilot (SP) transmission and it is studied in [19-25] for the case of a single-cell system. This method allows the use of the whole UL part of the coherence block for both pilots and data simultaneously. Improvements in bit-error-rate (BER) and mean squared error (MSE) of channel estimates are found in $[21,22]$ for SP in comparison to RP.

Recently, [23-25] have shown promising results in multicell Massive MIMO systems with SP. The main idea is that UEs transmit a linear combination of pilot and data symbols within the whole UL part of the coherence block. This allows the use of longer pilot sequences without having a pilot overhead. Hence, pilot contamination is reduced and the SE may be improved in comparison to RP. However, sending pilot and data symbols simultaneously leads to interference in the channel estimation process from 
data symbols. This degrades the estimation quality and creates correlation between channel estimates and data, which in turn, decreases the SE gain. Moreover, the use of longer pilots increases the computational complexity and power consumption which may eventually reduce the EE. All this may limit the practical gains of SP designs in terms of SE and EE.

In Paper A, the performance of SP in the UL of a multicell Massive MIMO system is evaluated and compared with RP. To this end, novel rigorous closedform rate expressions are derived with SP. This stands in contrast to prior works, [23, 25], which evaluate SP using approximate expressions of signalto-interference-plus-noise ratios (SINRs) and the MSEs of the channel estimates. In Paper B, RP and SP are compared in a multiobjective optimization framework where the UL and DL are jointly optimized for maximal sum SE.

\subsection{Hardware Design}

Most advantages of Massive MIMO are brought by the large number of antennas at the BS, however, this comes with a higher deployment cost and circuit power consumption. To implement this technology, the circuit cost and power consumption per antenna need to be significantly reduced in comparison to current BS's circuitry. This calls for low-complexity hardware at each antenna's radio frequency (RF) chain which is the chain of circuit components that are needed to transmit and receive data. However, reducing the hardware complexity introduces additional distortion into the system, degrading the SE. Fortunately, the large number of BS antennas is able to mitigate most of these effects by non-coherent distortion combining and significant gains in SE and EE can still be obtained with Massive MIMO [26,27]. Thus, there is a tradeoff in the number of BS antennas and hardware quality to achieve high SE and low power consumption.

An important source of distortion and power consumption in a Massive MIMO BS is the analog-to-digital converters (ADCs) which are responsible for sampling and quantizing the analog signals; and mapping them into digital signals. The number of quantization levels is given by the ADC bit resolution such that the distortion decreases whereas the power consumption and complexity increase with the ADC bit resolution. In [28-31] the selection of $\mathrm{ADC}$ bit resolution is studied to maximize performance in terms of BER, SE, and power consumption, showing that Massive MIMO can provide high SE with low-resolution ADCs. Here, low ADC bit resolution refers to values of up to 4-5 bits which is considerably lower than standard ADCs used in current BS deployments which have around 15 bits. The works [30,32] show that Massive MIMO can even operate with one-bit ADCs. However, it is not 
clear whether operating with such low hardware precision is preferable in terms of SE and EE.

The design of a wireless network entails weighing different performance metrics that are often mutually conflicting. In particular, this situation arises when maximal SE and minimal power consumption are desired. This tradeoff has been studied in $[33,34]$ for single-antenna and millimeter wave MIMO systems, respectively. In Paper C, the selection of the number of BS antennas and the ADC bit resolution is optimized for maximal SE under a power consumption constraint.

In all the aforementioned works, all RF chains have the same ADC bit resolution. In $[35,36]$, the tradeoff between hardware complexity and SE is studied in the case where some RF chains have high ADC bit resolution while the rest have low resolution. This approach is called mixed ADCs and significant gains in the SE have been reported in $[35,36]$ compared to using only low-resolution ADCs. However, the effect of channel estimation in MU-MIMO detection has not been considered and it has been assumed that switching mechanisms at sampling rate are available to adapt the ADC bit resolution of each antenna dynamically. This may be difficult to implement in practice for a massive number of RF chains. In [37], the authors consider the more practical scenario with mixed ADCs that remain static over time. The paper studies the optimal proportion of antennas with either high or low ADC bit resolution to maximize the SE under a power constraint. Numerical results indicate that using many BS antennas with only one-bit ADCs is the best approach. In Paper D, mixed ADCs are studied under imperfect channel estimation where the ADC bit resolution of each antenna can be set individually but remains static over time. Majorization theory $[38,39]$ is used to study the order preserving properties of the SE and the power consumption with respect to how unequal the ADC bit resolutions are across the BS antennas.

\subsection{Contributions of this Thesis}

This thesis analyzes alternative designs for a Massive MIMO system in terms of signal processing and hardware. The main idea is to conduct a rigorous analysis of these alternative designs and compare them with the baseline approaches to evaluate the potential gains (if any) that can be attained. The first alternative design is based on SP, where pilot and data symbols are sent simultaneously, instead of separately, to reduce pilot contamination and remove the pilot overhead. The results show that SP is able to reduce the pilot contamination effect at the expense of adding additional interfer- 
ence that limits the SE gain. The second alternative design is mixed ADCs, in which each BS antenna is allowed to have a different ADC bit resolution. The results show that having equal ADC bit resolution across the antenna array maximizes the SE and minimizes the power consumption, under a constraint on the total number of ADC bits.

This thesis is structured in two main parts: an introduction to the research topic and a collection of scientific papers. The introduction is meant to explain the main concepts, assumptions and insights found in the scientific papers and prior work. In Chapter 2, the basic theory and performance metrics for Massive MIMO are explained. The main ideas behind the alternative Massive MIMO designs including system model, main assumptions, performance evaluation, and insights are explained in Chapter 3. Finally, Chapter 4 summarizes the main contributions of this thesis and presents potential directions for future work.

The main ideas and topics researched in this thesis have emerged from the combined effort of the first author, supervisor and co-supervisors. The analysis and writing of the papers presented in this thesis have all been conducted by the first author with the careful guidance and advice from his supervisor and co-supervisors. The first author would like to acknowledge the contributions provided by the supervisor and co-supervisors through careful revision of manuscripts and analysis, as well as sharing their expert advice on review processes, research directions and general knowledge.

\subsection{Included Papers}

\section{Paper A: Spectral and Energy Efficiency of Superimposed Pilots in Uplink Massive MIMO}

Authored by: Daniel Verenzuela, Emil Björnson and Luca Sanguinetti Submitted to: IEEE Transactions on Wireless Communications.

This papers compares the performance of SP and RP in terms of SE and EE, for the UL of a multicell Massive MIMO network. A rigorous closedform rate expression with SP is derived assuming a simple linear processing method called maximum ratio combining (MRC). This formula provides valuable insights into identifying all the interference sources, their impact on the SE and their relationship with the other system parameters. Then, stochastic geometry is used to study the effect associated with intercell interference in a large practical network with an irregular BS deployment. Within this setting, a closed-form lower bound on the rates with SP averaged over 
the UEs' spatial distribution is derived. This provides powerful insights into the interplay of system parameters without requiring the use of heavy numerical simulations. Such lower bounds are then used to compute the EE of the network with both RP and SP taking into account the power consumed by the signal transmission and circuitry operation. Numerical results show that, when both methods are optimized, RP provides comparable SE and EE to SP in practical scenarios.

\section{Paper B: Joint UL and DL Spectral Efficiency Optimization of Superimposed Pilots in Massive MIMO}

Authored by: Daniel Verenzuela, Emil Björnson and Luca Sanguinetti

Published in Proceedings of: 2017 IEEE Global Communications Conference Workshops (GC Wkshps).

This work performs a comparative analysis of RP and SP in Massive MIMO considering the joint SE of UL and DL communications. A rigorous DL lower bound on the capacity with SP is derived, from which the different sources of interference are identified and an analytical comparison between RP and $\mathrm{SP}$ is offered. Then, multiobjective optimization theory is used to compare the joint UL and DL SE performance between RP and SP. Numerical results indicate that RP and SP give comparable SE when both methods are optimized.

\section{Paper C: Hardware Design and Optimal ADC Resolution for Uplink Massive MIMO Systems}

Authored by: Daniel Verenzuela, Emil Björnson and Michail Matthaiou Published in Proceedings of: 2016 IEEE Sensor Array and Multichannel Signal Processing Workshop (SAM).

This work focuses on the hardware design for the efficient operation of Massive MIMO systems. A closed-form UL achievable data rate expression is derived considering imperfect CSI and hardware impairments. An optimization problem is formulated to maximize the sum data rate subject to a constraint on the total power consumption. The optimization variables are the number of BS antennas and the level of hardware impairments per BS antenna. The solution manifests the fundamental tradeoff between the number of BS antennas and level of hardware impairments. For the case where the source of hardware impairments is the quantization distortion from ADCs, numerical results suggest that hardware quality corresponding to 4 or $5 \mathrm{ADC}$ bit resolution maximizes the SE. Moreover, by properly selecting the power consumption constraint, EE is also maximized for these ADC bit resolutions. 


\section{Paper D: Per-Antenna Hardware Optimization and Mixed Resolution ADCs in Uplink Massive MIMO}

Authored by: Daniel Verenzuela, Emil Björnson and Michail Matthaiou Published in Proceedings of: 2017 51st Asilomar Conference on Signals, Systems, and Computers.

This work studies the selection of per-antenna hardware quality in terms of $\mathrm{ADC}$ bit resolution. A new SE expression is derived for MRC and majorization theory is used to analyze the order preserving properties of the SE and the power consumption with respect to the per-antenna ADC bit resolutions. That is, given a fixed sum of ADC bits across the antenna array, is it preferable to use an equal-ADC over a mixed-ADC approach? The results show that having equal-resolution ADCs across the antenna array maximizes the $\mathrm{SE}$ and minimizes the power consumption.

\subsection{Excluded Papers}

The author of this thesis has also co-authored other papers that have not been included in this thesis because either they are condense versions of the included papers or they are outside the scope of this thesis. The excluded papers that are presented as follows:

\section{Condense versions}

D. Verenzuela, E. Björnson and L. Sanguinetti, "Spectral Efficiency of Superimposed Pilots in Uplink Massive MIMO Systems", in The Proceedings of IEEE Global Communications Conference (GLOBECOM), Singapore, Dec. 2017, pp. 1-6.

\section{Out-of-scope}

D. Verenzuela, E. Björnson and L. Sanguinetti, "Optimal Design of Wireless Networks for Broadband Access with Minimum Power Consumption", in The Proceedings of IEEE International Conference on Communications (ICC), Kuala Lumpur, Malaysia, May. 2016, pp. 1-6.

A. Pizzo, D. Verenzuela, L. Sanguinetti and E. Björnson, "Network Deployment for Maximal Energy Efficiency in Uplink with Zero-Forcing", in The Proceedings of IEEE Global Communications Conference (GLOBECOM), Singapore, Dec. 2017, pp. 1-7. 
A. Pizzo, D. Verenzuela, L. Sanguinetti and E. Björnson, "Network Deployment for Maximal Energy Efficiency in Uplink with Multislope pathloss", Submitted to IEEE Transactions on Green Communications and Networking, Dec. 2017. [Online]. Available: https://arxiv.org/abs/1801.00043 


\section{Chapter 2}

\section{Massive MIMO}

This chapter introduces the baseline system model, main assumptions and performance metrics used for analyzing Massive MIMO systems in this thesis. First, the communication channel is defined. Second, basic properties of Massive MIMO channels are explained. Third, the process of channel estimation is introduced. Fourth, main results on the capacity analysis are presented. Finally, the main performance metrics used in this thesis are introduced, that is, SE, EE and power consumption. In addition, a summary of the main benefits and characteristics of Massive MIMO is provided.

\subsection{Communication Channel}

The communication channel is the input-output relationship between the transmitter and receiver. In wireless communications, electromagnetic waves are used to carry the information from the transmitter to the receiver. These waves propagate through the air encountering obstacles that reflect, diffract, and attenuate them. These phenomena change the amplitude and phase of the propagating signal, such that, in the far field, the received signal is given by a linear combination of attenuated and rotated versions of the transmitted signal that have taken different paths. Since the UEs and obstacles in a cellular network are mobile, the communication channel varies in time and frequency, which is referred to as a fading channel [40].

The radio frequencies that can be used for cellular wireless communications are set by governmental institutions. Thus, the signals need to be transmitted at a specific carrier frequency $f_{c}$ over a set bandwidth $B_{\mathrm{W}}$ as shown in Figure 1a. However, the signal processing is usually done at baseband (i.e., $f_{c}=0$ ) to simplify the implementation and then up/down converted to the desired carrier frequency. Since the physical signal that is transmitted is 


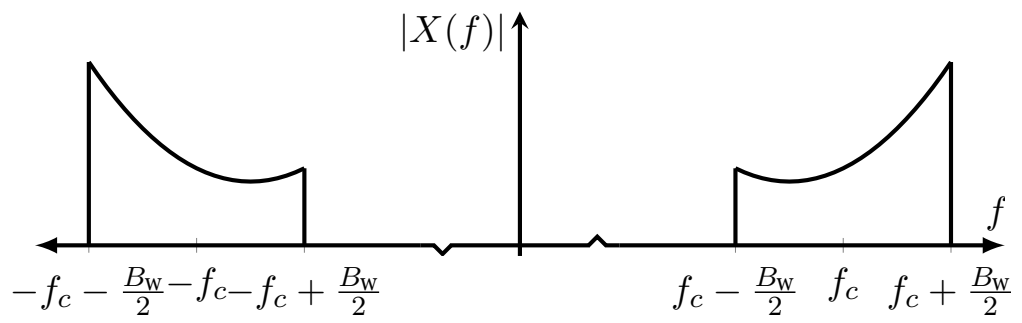

(a) Real-valued signal transmitted at a carrier frequency $f_{c}$.

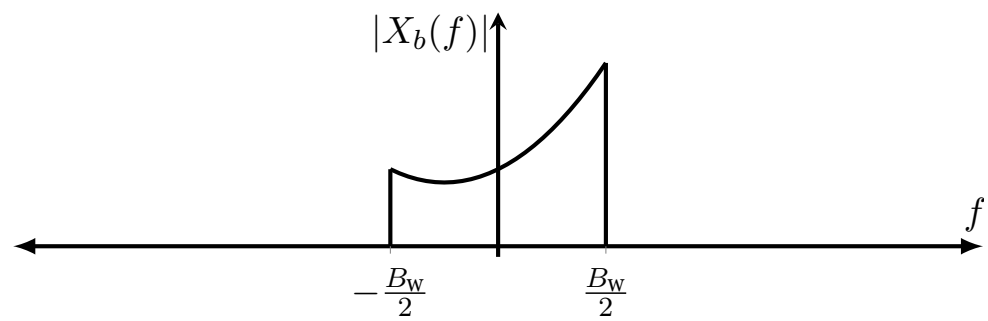

(b) Complex baseband equivalent signal.

Figure 1: Magnitude spectrum of passband and baseband signal.

real valued, its spectrum is an even function (as shown in Figure 1a) and all the signal information is contained, in the bandwidth $B_{\mathrm{W}}$, either at positive or negative frequencies. Thus, the wireless channel, in the far field, can be described by a linear time varying system with a baseband representation (see Figure 1b) given by [40]

$$
y(t)=\sum_{i} a_{i}(t, f) x\left(t-\tau_{i}(t, f)\right)
$$

where $x(t)$ and $y(t)$ are the complex baseband transmitted and received signals at time $t$, respectively. The attenuation and phase shift of the channel in the $i^{t h}$ path is $a_{i}(t, f)$ and $\tau_{i}(t, f)$, respectively, and are functions of both time $t$ and frequency $f$. However, since the carrier frequency is usually much larger than the bandwidth, $f_{c} \gg B_{\mathrm{W}}$, the changes in $a_{i}(t, f)$ and $\tau_{i}(t, f)$ with respect to $f$, within $B_{\mathrm{W}}$, can be neglected. Thus, $a_{i}(t, f)=a_{i}(t)$ and $\tau_{i}(t, f)=\tau_{i}(t)$ are taken as the attenuation and phase shift of the channel in the $i^{t h}$ path at $f \approx f_{c}$. It is worth to stress that even though the effect of each path can be considered to be independent of frequency, the aggregated effect of the channel is indeed frequency dependent.

In general terms, the effect of the wireless channel can be classified in two parts: 


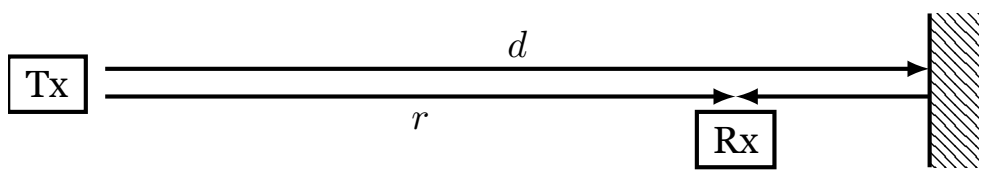

Figure 2: Two path propagation model. Tx and Rx stand for transmitter and receiver, respectively.

Large-scale fading (LSF). This is caused by obstacles and movements that are orders of magnitude larger than the wavelength of the propagating signals. This effect is mainly responsible for attenuating the propagating signals due to pathloss, and shadowing from large obstacles. The LSF is considered to vary slowly in time (in the order of seconds or minutes depending on UE mobility) with respect to the communication delay constraints (in the order of milliseconds). Moreover, since $B_{\mathrm{W}}$ is usually far lower than $f_{c}$, the attenuation changes from the LSF can be considered to be frequency independent.

Small-scale fading (SSF). This is caused by small obstacles and movements in the same order of magnitude as the wavelength of the propagating signals. This effect causes the signal to scatter, taking different paths, and resulting in phase shifts that may add constructively or destructively at the receiver, which is called multipath fading. The phase and amplitude changes occur in a fast time scale (in the order of milliseconds or less) and are frequency dependent due to the relative movements of the transmitter, receiver and obstacles.

The time and frequency variations of the SSF are orders of magnitude faster than the LSF, and for the design of communication systems, it is important to understand how these fast variations behave. To gain some insights into the physical meaning of the SSF effects, consider the scenario in Figure 2 where the transmitted signal is perfectly reflected by an infinite wall creating two paths for the received signal. For simplicity, assume that the difference in distance between the paths is small enough so that the attenuation is the same and the transmission power is normalized such that $a_{0}(t)=a_{1}(t)=1$. Let $x(t)=\cos (2 \pi f t)$, then the received signal is

$$
\begin{aligned}
y(t) & =\cos \left(2 \pi f\left(t-\tau_{1}\right)\right)-\cos \left(2 \pi f\left(t-\tau_{2}\right)\right) \\
& =\cos \left(2 \pi f\left(t-\frac{r}{c}\right)\right)-\cos \left(2 \pi f\left(t-\frac{2 d-r}{c}\right)\right)
\end{aligned}
$$

where $\tau_{1}=r / c$ and $\tau_{2}=(2 d-r) / c$ are the time delays of the signal from the first and second path, respectively. The waves travel at the speed of light, 
denoted by $c$, so that the phase shift at a distance $r$ is the travel time of the wave, that is $r / c$. Note that the minus sign for the second path is introduced by the reflection upon the wall [40].

The fast time variations are mainly due to the Doppler shifts introduced by relative movements of the transmitter, receiver, or obstacles. In the example shown in Figure 2, consider that the transmitter is fixed and the receiver is moving in the direction of propagation (towards the wall). The position of the receiver at time $t$ is given by $r=r_{0}+v t$ where $r_{0}$ is the initial position and $v>0$ is the constant velocity of the receiver, the received signal is then

$$
\begin{aligned}
y(t) & =\cos \left(2 \pi f\left(\left(1-\frac{v}{c}\right) t-\frac{r_{0}}{c}\right)\right)-\cos \left(2 \pi f\left(\left(1+\frac{v}{c}\right) t-\frac{2 d-r_{0}}{c}\right)\right) \\
& =2 \sin \left(2 \pi f\left(t-\frac{d}{c}\right)\right) \sin \left(2 \pi f\left(\frac{v t}{c}+\frac{r_{0}-d}{c}\right)\right) .
\end{aligned}
$$

The Doppler effect causes the received signals from the two paths to have frequency shifts of $D_{1}=-f v / c$ and $D_{2}=f v / c$, respectively. The difference between the Doppler shifts is called the Doppler spread and is given by $D_{s}=D_{2}-D_{1}$. The Doppler spread dictates the time period of the amplitude variations and for this example the period is $2 / D_{s}$, which corresponds to the time period of the second sinus in (5). In a more general scenario, where there are multiple paths with different Doppler shifts, the Doppler spread is defined as $D_{s}=\max _{i j}\left|D_{i}-D_{j}\right|$. In such cases, the aggregated effect of multiple Doppler shifts is often modeled as a realization of a random variable. The time interval between significant changes of the channel, in an order of magnitude sense, is called the coherence time and is given by [40]

$$
T_{c}=\frac{1}{4 D_{s}} .
$$

Thus, within this time period the channel can be approximated by a timeinvariant system.

The variations in frequency are caused by the time difference between paths that the received signal has taken. In the example from Figure 2, the phase difference in the signal received from the two paths, shown in (4), is $2 \pi f T_{d}+\pi$ where $T_{d}=\tau_{2}-\tau_{1}$ is the time difference between the paths called the delay spread. By taking the Fourier transform of (4), the magnitude of the frequency response of the system can be computed as

$$
\left|\frac{Y(f)}{X(f)}\right|=\left|\mathrm{e}^{-j 2 \pi f \tau_{1}}-\mathrm{e}^{-j 2 \pi f \tau_{2}}\right|=2\left|\sin \left(\pi f T_{d}\right)\right|
$$

where the frequency period of these variations, that is the period of the sinusoid in (7) in terms of $f$, is $2 / T_{d}$. In a more general case where there 
are several paths with different delays, the delay spread can be defined as $T_{d}=\max _{i, j}\left|\tau_{i}-\tau_{j}\right|$. In this case, the aggregated effect of the delays introduced by many paths is often modeled by a random variable. The bandwidth interval in which the channel undergoes significant changes, in an order of magnitude sense, is called the coherence bandwidth and is given by [40]

$$
B_{c}=\frac{1}{2 T_{d}} .
$$

Thus, within this frequency period the frequency response of the channel can be considered to be approximately flat.

From the results in (6) and (8), if the received signal is observed during a coherence time and within a coherence bandwidth, the channel can be approximated by a linear time invariant system with flat frequency response. This time-frequency block is called the coherence block.

Based on the sampling theorem [41], a continuous time complex-valued signal with spectrum contained in a finite bandwidth within $\left[-B_{c} / 2, B_{c} / 2\right]$, can be represented by a discrete time complex-valued signal via pulse amplitude modulation. The discrete time complex-valued signal is composed of complex samples taken at the sampling rate of $f_{s} \geq B_{c}$. Then, a signal that is contained in a coherence time $T_{c}$ and a coherence bandwidth $B_{c}$ can be represented by $\tau_{c}=T_{c} B_{c}$ complex samples which constitute a coherence block. These complex samples are also referred to as the degrees of freedom or channel uses that are available in the coherence block. Finally, the discrete time complex basedband representation of (3) in a coherence block is

$$
y[n]=h \sqrt{\rho} x[n] \in \mathbb{C} \quad \text { for } \quad n \in\left\{1, \ldots, \tau_{c}\right\}
$$

where $x[n]$ and $y[n]$ are the discrete time transmitted and received signals, respectively, obtained by sampling $x(t)$ and $y(t)$ at a rate $f_{s}=B_{c}$. This is called the Nyquist sampling rate and it is going to be used throughout this thesis. The aggregated effect of the channel is given by $h \in \mathbb{C}$. The term $\rho$ is the transmission energy per symbol and since the sampling frequency is the same as the bandwidth, the transmission power is $\rho B_{c}$. In this thesis, there has been some abuse of notation and the term $\rho$ is sometimes referred to as the transmission power or transmission power per symbol. Note that the latter is a wrong definition and hence the clarification.

\subsection{Properties of Massive MIMO Channels}

To explain how the channel properties affect the communication between the transmitter and receiver, consider a single-input multiple-output (SIMO) 
system where the receiver is equipped with $M$ antennas so that $\mathbf{h} \in \mathbb{C}^{M \times 1}$ corresponds to the communication channel. Assume for simplicity that the coherence block is infinite so that the channel can be considered to be deterministic and perfectly known by the transmitter and the receiver. Thus, the received signal in one arbitrary symbol of the coherence block is

$$
\mathbf{y}=\mathbf{h} \sqrt{\rho} x+\mathbf{n} \in \mathbb{C}^{M \times 1} .
$$

The transmitted information-bearing signal is represented by a zero-mean random variable $x \in \mathbb{C}$ such that $\mathbb{E}\left\{|x|^{2}\right\}=1$ and $\rho$ is the average transmitted energy per symbol. The receiver noise is distributed as $\mathbf{n} \sim \mathcal{C N}\left(\mathbf{0}, \mathbf{I}_{M} \sigma^{2}\right)$ with $\sigma^{2}$ corresponding to the average noise energy per symbol. The maximum achievable performance of this system is well defined by the Shannon's capacity $[7,8,40]$ and it is given by

$$
C_{\text {SIMO }}=\log _{2}\left(1+\frac{\rho\|\mathbf{h}\|^{2}}{\sigma^{2}}\right)[\mathrm{bpcu}] .
$$

The capacity indicates the maximum rate of information between transmitter and receiver (here expressed in bits per channel use [bpcu]) that can be achieved with an arbitrarily low error probability (this is explained with more detail in Section 2.4). It can be seen from (11) that the capacity increases logarithmically with the total energy, $\|\mathbf{h}\|^{2}$, collected by the receiver antenna array which in turn increases with the number of antennas. ${ }^{1}$

Now consider the UL of a MU-MIMO system with two UEs denoted as $\mathrm{UE}_{1}$ and $\mathrm{UE}_{2}$. The BS is equipped with $M$ antennas so that $\mathbf{h}_{1}, \mathbf{h}_{2} \in \mathbb{C}^{M \times 1}$ correspond to the channels between the $\mathrm{BS}$ and $\mathrm{UE}_{1}, \mathrm{UE}_{2}$, respectively. As before, let the channels be deterministic and perfectly known. The received signal in one arbitrary symbol of the coherence block is

$$
\mathbf{y}=\mathbf{h}_{1} \sqrt{\rho} x_{1}+\mathbf{h}_{2} \sqrt{\rho} x_{2}+\mathbf{n} \in \mathbb{C}^{M \times 1} .
$$

The transmitted signals of $\mathrm{UE}_{1}$ and $\mathrm{UE}_{2}$ are the zero-mean random variables $x_{1}, x_{2} \in \mathbb{C}$ such that $\mathbb{E}\left\{\left|x_{1}\right|^{2}\right\}=1$ and $\mathbb{E}\left\{\left|x_{2}\right|^{2}\right\}=1$, respectively. In this case, the sum capacity is given by $[7,40]$

$$
\begin{aligned}
C_{\text {SUM }} & =\log _{2}\left(\operatorname{det}\left(\mathbf{I}_{M}+\frac{\rho}{\sigma^{2}}\left(\mathbf{h}_{1} \mathbf{h}_{1}^{H}+\mathbf{h}_{2} \mathbf{h}_{2}^{H}\right)\right)\right)[\text { bpcu }] \\
& =\log _{2}\left(\left(1+\frac{\rho}{\sigma^{2}}\left\|\mathbf{h}_{1}\right\|^{2}\right)\left(1+\frac{\rho}{\sigma^{2}}\left\|\mathbf{h}_{2}\right\|^{2}\right)-\frac{\rho^{2}}{\sigma^{4}}\left|\mathbf{h}_{1}^{H} \mathbf{h}_{2}\right|^{2}\right)[\mathrm{bpcu}]
\end{aligned}
$$

\footnotetext{
${ }^{1}$ This holds in cases where the aperture of the array increases proportionally with the number of antennas, that is, when $\|\boldsymbol{h}\|^{2}$ grows linearly with $M$.
} 
and is maximized for channels that are orthogonal (i.e., $\mathbf{h}_{1}^{H} \mathbf{h}_{2}=0$ ) which yields

$$
C_{\mathrm{SUM}}=\log _{2}\left(1+\frac{\rho}{\sigma^{2}}\left\|\mathbf{h}_{1}\right\|^{2}\right)+\log _{2}\left(1+\frac{\rho}{\sigma^{2}}\left\|\mathbf{h}_{2}\right\|^{2}\right)[\mathrm{bpcu}] .
$$

This result corresponds to the sum of the capacity in (11) of two individual SIMO systems. Therefore, a desirable property of MU-MIMO channels is

$$
\mathbf{h}_{i}^{H} \mathbf{h}_{j}= \begin{cases}0 & \text { for } i \neq j \\ \left\|\mathbf{h}_{i}\right\|^{2} & \text { for } i=j\end{cases}
$$

which is called favorable propagation.

In cellular networks, the channel varies in time and frequency and the coherence block approximation would only be reasonable for a limited number of samples $\tau_{c}$ that are given by the propagation environment. Thus, the assumption of deterministic or perfectly known channels is dropped for the rest of this thesis. As seen in Section 2.1, the LSF effect depends on the UE's mobility and usually changes slowly compared to the communication delay constraints, thus it is easy to track, and in this thesis, is considered to be fixed and known. On the other hand, the SSF introduces variations in the amplitude and phase of transmitted signals that are in the same order, or faster than the communication delay constraints. Since the propagation environment changes continuously, it is hard to track each path that the transmitted signal takes to evaluate the channel deterministically. Thus, a stochastic model is used to gain insights into the performance of the system under fading channels.

The channel between $\mathrm{UE}_{i}$ and the $\mathrm{BS}$ is then modeled as a random vector distributed as $\mathbf{h}_{i} \sim \mathcal{C N}\left(\mathbf{0}, \mathbf{I}_{M} \beta_{i}\right)$ having independent realizations across different coherence blocks but remaining constant in each block. The effect of the SSF is comprised in the realizations of $\mathbf{h}_{i}$ and the LSF effect is given by the variance $\beta_{i} \geq 0$ which is assumed to be fixed and known. This is called the block fading model with independent identically distributed (i.i.d.) Rayleigh fading and it is adopted throughout this thesis.

This channel model provides a good approximation of propagating environments with rich scattering where the signals between the UEs and the BS take many different paths and arrive in many different directions. In [42], it has been shown that the rate performance predicted under the i.i.d. Rayleigh fading assumption, is close to that under practical measured channels with non-line-of-sight and spatially spread UEs. However, this model does not take into account the spatial correlation between the signals received by (or transmitted from) different BS antennas which may add inter- 
esting additional perspectives to the results provided in this thesis. A detailed analysis of the spatial correlation effects in Massive MIMO is done in [8].

Note that in general, favorable propagation does not hold in real propagation environments. In particular, for i.i.d. Rayleigh fading channels, the inner product between two channel vectors is a random variable and favorable propagation does not hold either. However, in Massive MIMO the main assumption is that the number of BS antennas $M$ is large, and in this case, two channel properties arise thanks to the law of large numbers:

Asymptotic favorable propagation which occurs when the inner product, normalized by $M$, between channels from (or to) different UEs become asymptotically orthogonal as $M$ increases. That is for $i \neq j$

$$
\frac{\mathbf{h}_{i}^{H} \mathbf{h}_{j}}{M} \stackrel{\text { a. s. }}{\longrightarrow} 0 \quad \text { as } \quad M \rightarrow \infty
$$

where a. s. denotes almost sure convergence.

Channel hardening which means that the squared norm of the channel normalized by $M$ becomes deterministic as $M$ increases, that is,

$$
\frac{\left\|\mathbf{h}_{i}\right\|^{2}}{M}-\beta_{i} \stackrel{\text { a.s. }}{\longrightarrow} 0 \quad \text { as } \quad M \rightarrow \infty .
$$

These properties hold for a wide range of Massive MIMO channels and simplify its implementation [7,8], in particular, they hold for i.i.d. Rayleigh channels. Thanks to asymptotic favorable propagation, the channels between the BS and different UEs are easier to separate in the spatial domain as $M$ increases. In the case when linear signal processing is used to detect the data symbols, the asymptotic favorable propagation effect reduces the interference between UEs as $M$ grows large (as an example, see the last term inside the logarithm in (13)). On the other hand, channel hardening allows the power control to be independent from the SSF variations and it makes the channel estimation in the DL easier. Thus, these channel properties allow simple linear processing methods to perform well, providing high SE to several UEs simultaneously with low complexity. These effects are explained with further detailed in Section 2.5.

\subsection{Channel Estimation}

In this thesis, pilot based channel estimation is considered to conduct coherent signal processing. Pilot sequences are sent by the UEs in the UL from 


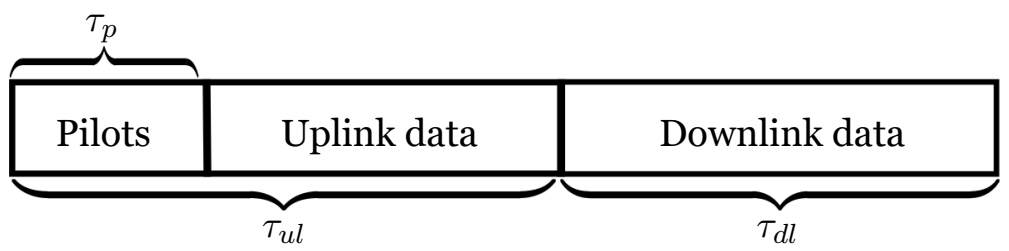

Figure 3: TDD transmission protocol of the baseline Massive MIMO system (also referred to as RP).

which the BS can estimate the channels. A TDD protocol is assumed and time reciprocity is exploited so that the UL channel estimates can be used to process signals in the DL. Figure 3 illustrates a TDD communication protocol where $\tau_{u l}$ and $\tau_{d l}$ are the number of symbols in the coherence block used for UL and DL, respectively, such that $\tau_{c}=\tau_{u l}+\tau_{d l}$.

Consider the UL of a multicell MU-MIMO system where each BS deploys $M$ antennas and serves $K$ single-antenna UEs in its cell. Denote as $\mathrm{BS}_{l}$ the BS $l \in \Phi$, where $\Phi$ is a set with the indices of all BSs in the network, and $\mathrm{UE}_{l k}$ the UE $k$ that is served by $\mathrm{BS}_{l}$. The channel between the $M$ antennas of $\mathrm{BS}_{l}$ and $\mathrm{UE}_{l k}$ is defined as $\mathbf{h}_{l l k} \sim \mathcal{C N}\left(\mathbf{0}, \beta_{l l k} \mathbf{I}_{M}\right)$, where $\beta_{l l k}$ accounts for the LSF. As shown in Figure 3, $\tau_{p}$ out of the $\tau_{u l}$ symbols in the UL are used to perform channel estimation. This corresponds to the baseline operation of Massive MIMO, which is referred to as RP. For ease of notation, the analysis is focused on $\mathrm{BS}_{0}$ and $\mathrm{UE}_{0 k} .{ }^{2}$ The pilot sequence used by $\mathrm{UE}_{0 k}$ is $\phi_{0 k} \in \mathbb{C}^{\tau_{p} \times 1}$ with elements having unit modulus, that is, $\left|\left[\phi_{0 k}\right]_{j}\right|=1 \forall j \in\left\{1, \ldots, \tau_{p}\right\}$. The pilots assigned to the UEs within the same cell are selected to be orthogonal, such that

$$
\phi_{0 k}^{H} \phi_{0 i}= \begin{cases}\tau_{p} & \text { for } k=i \\ 0 & \text { for } k \neq i\end{cases}
$$

This means that only $K \leq \tau_{p}$ UEs can be served simultaneously in one cell. Notice that only $\tau_{p}$ mutually orthogonal pilot sequences are available in the network, such that $\phi_{l i} \in \mathcal{U}_{\tau_{p}}$, where $\mathcal{U}_{\tau_{p}}$ is the set of all mutually orthogonal pilots in the system with cardinality $\tau_{p}$. Since the total number of UEs in all cells is usually larger than $\tau_{p}$, pilots need to be reused among cells. The set of indices containing the UEs that have the same pilot as $\mathrm{UE}_{0 k}$, including $\mathrm{UE}_{0 k}$, is defined as

$$
\mathcal{P}_{0 k}=\left\{(l, i): \phi_{0 k}^{H} \phi_{l i} \neq 0\right\} .
$$

\footnotetext{
${ }^{2}$ Since the spatial distribution of BSs and UEs is kept unspecified, this notation does not influence the analysis.
} 
The selection of the UEs that belong to this set depends on the pilot allocation scheme which is left undefined, for now, to obtain a more general result.

The received signal at the $M$ antennas of $\mathrm{BS}_{0}$ over the $\tau_{p}$ samples of the coherence block used for pilots is

$$
\mathbf{Z}_{0}=\sum_{l \in \Phi} \sum_{i=1}^{K} \mathbf{h}_{0 l i} \sqrt{q_{l i}^{\mathrm{RP}}} \phi_{l i}^{T}+\mathbf{N}_{0} \in \mathbb{C}^{M \times \tau_{p}}
$$

where $\mathbf{N}_{0}$ is the noise matrix with elements independently distributed as $\left[\mathbf{N}_{0}\right]_{m j} \sim \mathcal{C N}\left(0, \sigma^{2}\right) \forall m \in\{1, \ldots, M\}, \forall j \in\left\{1, \ldots, \tau_{p}\right\}$, and with average noise energy per symbol $\sigma^{2}$. The transmission energy per pilot symbol sent by $\mathrm{UE}_{l i}$ is $q_{l i}^{\mathrm{RP}}$. The channel estimates are obtained by first performing a despreading operation on $\mathbf{Z}_{0}$ which yields the observation vector

$$
\mathbf{z}_{0 k}=\mathbf{Z}_{0} \frac{\phi_{0 k}^{*}}{\sqrt{\tau_{p}}}=\sum_{(l, i) \in \mathcal{P}_{0 k}} \mathbf{h}_{0 l i} \sqrt{q_{l i}^{\mathrm{RP}} \tau_{p}}+\mathbf{n}_{0} \in \mathbb{C}^{M \times 1}
$$

where $\mathbf{n}_{0} \sim \mathcal{C N}\left(\mathbf{0}, \mathbf{I}_{M} \sigma^{2}\right)$ is the projection of the noise matrix $\mathbf{N}_{0}$ on to $\phi_{0 k}^{*} / \sqrt{\tau_{p}}$. Second, the minimum MSE (MMSE) channel estimate of $\mathbf{h}_{00 k}$ is computed based on the observations $\mathbf{z}_{0 k}$. This result is summarized in the following lemma.

Lemma 1. The MMSE estimate of $\mathbf{h}_{00 k}$ based on $\mathbf{z}_{0 k}$ is

$$
\hat{\mathbf{h}}_{00 k}=\frac{\gamma_{0 k}}{\sqrt{q_{0 k}^{\mathrm{RP}} \tau_{p}}} \mathbf{z}_{0 k}
$$

where

$$
\gamma_{0 k}=\frac{q_{0 k}^{\mathrm{RP}} \tau_{p} \beta_{00 k}}{\sum_{(l, i) \in \mathcal{P}_{0 k}} q_{l i}^{\mathrm{RP}} \tau_{p} \beta_{0 l i}+\sigma^{2}} .
$$

The estimation error is defined as $\tilde{\mathbf{h}}_{00 k}=\mathbf{h}_{00 k}-\hat{\mathbf{h}}_{00 k}$. The covariance matrices of the channel estimates and the estimation errors are

$$
\begin{aligned}
& \mathbb{E}\left\{\hat{\mathbf{h}}_{00 k} \hat{\mathbf{h}}_{00 k}^{H}\right\}=\gamma_{0 k} \beta_{00 k} \mathbf{I}_{M}, \\
& \mathbb{E}\left\{\tilde{\mathbf{h}}_{00 k} \tilde{\mathbf{h}}_{00 k}^{H}\right\}=\left(1-\gamma_{0 k}\right) \beta_{00 k} \mathbf{I}_{M},
\end{aligned}
$$

respectively.

Proof. It follows from computing the mean of the channel $\mathbf{h}_{00 k}$ conditioned on the observations $\mathbf{z}_{0 k}$. This result is based on applying standard MMSE estimation techniques [43, Ch. 15.8] to the problem at hand and it can be found in $[7,8]$. 
From this lemma, there are two key points the are important to mention:

\section{Estimation quality improves with longer pilots.}

The covariance matrix of the channel estimates depends on $\tau_{p}$ through $\gamma_{0 k}$. In (20), it can be seen that as $\tau_{p}$ increases the effect of the noise vanishes, thus $\gamma_{0 k}$ is an increasing function of $\tau_{p}$. Moreover, as $\tau_{p}$ increases, there are more pilots available which means that they can be reused further apart in the network. This decreases the number of UEs in the set $\mathcal{P}_{0 k}$, reducing the pilot interference (see the denominator of (20)). In the extreme case when $\tau_{p}$ is so large that all UEs in the network can have an orthogonal pilot sequence, and the noise power is negligible compared to $q_{0 k}^{\mathrm{RP}} \tau_{p} \beta_{00 k}$, it follows that $\gamma_{0 k} \rightarrow 1$. However, in practice $\tau_{p}$ is limited by the coherence block size which is set by the propagation environment.

\section{Channel estimates and errors are statistically independent under Gaussian observations.}

For MMSE estimation, the estimates are uncorrelated with the estimation errors by design. In the case where the observations are Gaussian, as it is for $\mathbf{z}_{0 k}$ in (18), the MMSE estimates are linear in the observations which means that they coincide with the linear MMSE (LMMSE) estimates. In addition, the estimates and errors are jointly Gaussian random variables that are uncorrelated, and therefore, they are also statistically independent. This fact is commonly used in literature to calculated closed form expressions of lower bounds on the capacity $[7,8]$. However, notice that in cases where the observations are not Gaussian the estimates and errors remain uncorrelated but are not statistically independent. This fact plays an important role in the performance evaluation of Massive MIMO with SP, and under hardware impairments. This is explained with more detail in Chapter 3.

\subsection{Capacity Analysis}

To evaluate the performance of a communication system, a key metric is the maximum rate of information that can be conveyed from the transmitter to the receiver in a reliable way. An achievable rate is defined as an information rate (here measured in [bpcu]) that can be achieved by the communication between a transmitter and a receiver with an error probability that can be made arbitrarily low as the block-length of the coded information increases $[7,8,40]$. The capacity is defined as the maximum achievable rate 
that is reached when the block-length of the coded information goes to infinity. In other words, the capacity gives the best information rate that can be achieved error-free.

The capacity of a single cell MU-MIMO system under a fast fading channel and white Gaussian noise is known for the case where the receiver has perfect channel knowledge [44]. However, when the receiver does not known the channel or there is non-Gaussian interference, the capacity is unknown. In these cases, there are expressions for lower bounds on the capacity that can serve as performance metrics and are used throughout this thesis.

Recall that from the sampling theorem, a signal contained within a bandwidth $B_{\mathrm{W}}[\mathrm{Hz}]$ and a time $T_{c}$ [s], can be represented by $B_{\mathrm{W}} T_{c}$ complex samples. Thus, if $B_{\mathrm{W}}$ complex samples per second are used for communicating information within a bandwidth $B_{\mathrm{W}}$, an achievable rate of $\mathrm{R}$ [bpcu] is equivalent to an SE of R [bit/s/Hz]. For the MU-MIMO system model introduced in Section 2.3, the received data signal at $\mathrm{BS}_{0}$ in an arbitrary symbol of the coherence block is

$$
\mathbf{y}_{0}=\sum_{l \in \Phi} \sum_{i=1}^{K} \mathbf{h}_{0 l i} \sqrt{\rho_{l i}^{\mathrm{RP}}} s_{l i}+\mathbf{n}_{0} \in \mathbb{C}^{M \times 1}
$$

where $\mathbf{n}_{0} \sim \mathcal{C N}\left(\mathbf{0}, \mathbf{I}_{M} \sigma^{2}\right)$ is the noise vector with $\sigma^{2}$ as the average noise energy per symbol and $\rho_{l i}^{\mathrm{RP}}$ as the average transmission energy per data symbol from $\mathrm{UE}_{l i}$. The data symbol transmitted by $\mathrm{UE}_{l i}$ is $s_{l i}$ and it is assumed to be zero mean and have unit variance. To perform coherent detection, linear signal processing is typically considered in Massive MIMO since it offers linear computational complexity in $M$. Thus, $\mathrm{BS}_{0}$ multiplies the received signal with the combining matrix $\mathbf{A}=\left[\mathbf{a}_{1}, \ldots, \mathbf{a}_{K}\right]$ to obtain $K$ equivalent single-input single-output (SISO) systems corresponding to the $K$ multiplexed UEs. For $\mathrm{UE}_{0 k}$ the equivalent SISO received signal is then used as an estimate of the transmitted data symbol which is given by

$$
\begin{aligned}
\hat{s}_{0 k}= & \mathbf{a}_{k}^{H} \mathbf{y}_{0} \\
= & \underbrace{\mathbf{a}_{k}^{H} \mathbf{h}_{00 k} \sqrt{\rho_{0 k}^{\mathrm{RP}}} s_{0 k}}_{\text {Desired signal }}+\underbrace{\sum_{\substack{i=1 \\
i \neq k}}^{K} \mathbf{a}_{k}^{H} \mathbf{h}_{00 i} \sqrt{\rho_{0 i}^{\mathrm{RP}}} s_{0 i}}_{\text {Intracell interference }}+\underbrace{\sum_{l \in \Phi \backslash 0} \sum_{i=1}^{K} \mathbf{a}_{k}^{H} \mathbf{h}_{0 l i} \sqrt{\rho_{l i}^{\mathrm{RP}}} s_{l i}}_{\text {Intercell interference }}+\underbrace{\mathbf{a}_{k}^{H} \mathbf{n}_{0}}_{\text {Noise }} .
\end{aligned}
$$

To combine the received signals from each BS antenna coherently, the selection of $\mathbf{a}_{k}$ is based on the channel estimates that were obtained in Section 2.3. The capacity of the equivalent SISO system in (24) is unknown since the 
channels are unknown and the intercell interference is non-Gaussian. A standard lower bound on the capacity can be obtained by applying the so called use-and-then-forget technique [7], in which the channel estimates are used for constructing $\mathbf{a}_{k}$ but are then neglected when computing the capacity bound. Thus, the data estimates can be rewritten as

$$
\hat{s}_{0 k}=\underbrace{\mathbb{E}\left\{\hat{s}_{0 k} s_{0 k}^{*}\right\}}_{\begin{array}{c}
\text { Deterministic } \\
\text { effective channel }
\end{array}} s_{0 k}+\underbrace{\hat{s}_{0 k}-\mathbb{E}\left\{\hat{s}_{0 k} s_{0 k}^{*}\right\} s_{0 k}}_{\begin{array}{c}
\text { Non-Gaussian } \\
\text { effective noise }
\end{array}}
$$

and a lower bound on the capacity is given by $[7,8]$

$$
\begin{aligned}
\mathbf{R}_{0 k} & =\left(1-\frac{\tau_{p}}{\tau_{u l}}\right) \log _{2}\left(1+\operatorname{SINR}_{0 k}\right), \\
\operatorname{SINR}_{0 k} & =\frac{\left|\mathbb{E}\left\{\hat{s}_{0 k} s_{0 k}^{*}\right\}\right|^{2}}{\operatorname{Var}\left\{\hat{s}_{0 k}-\mathbb{E}\left\{\hat{s}_{0 k} s_{0 k}^{*}\right\} s_{0 k}\right\}}, \\
& =\frac{\left|\mathbb{E}\left\{\mathbf{a}_{k}^{H} \mathbf{h}_{00 k}\right\}\right|^{2} \rho_{0 k}^{\mathrm{RP}}}{\operatorname{Var}\left\{\mathbf{a}_{k}^{H} \mathbf{h}_{00 k}\right\} \rho_{0 k}^{\mathrm{RP}}+\sum_{l \in \Phi} \sum_{i=1}^{K} \mathbb{E}\left\{\left|\mathbf{a}_{k}^{H} \mathbf{h}_{0 l i}\right|^{2}\right\} \rho_{l i}^{\mathrm{RP}}+\mathbb{E}\left\{\left|\mathbf{a}_{k}^{H} \mathbf{n}_{0}\right|^{2}\right\}},
\end{aligned}
$$

where the pre-log factor in (26) corresponds to the cost of using $\tau_{p}$ samples for channel estimation. ${ }^{3}$ The result in (27) holds for any SISO system with a deterministic effective channel and non-Gaussian effective noise as shown in (25). The effective SINR in (28) applies for linear processing and it holds for any choice of $\mathbf{a}_{k}$ or distribution of the channels $\mathbf{h}_{0 l i}$. The performance given by this bound may underestimate the real performance that can be achieved since the information available from the channel estimates is neglected when computing the bound itself. However, thanks to the effect of channel hardening, this bound tightens as $M$ grows large, which makes it a suitable metric to evaluate the performance of Massive MIMO [7]. In addition, this bound allows for a tractable analysis that results in closed-form, or semi closed-form, rate expressions that provide key insights into the system performance. There are other bounds that are closer to the capacity and can be used to evaluate other scenarios where, for instance, channel hardening does not hold.

To select the combining matrix, a standard choice that further simplifies the implementation is $\mathbf{a}_{k}=\hat{\mathbf{h}}_{00 k}$ which is called maximum ratio combining

\footnotetext{
${ }^{3}$ The result in (26) corresponds to the UL, so that $\tau_{c}=\tau_{u l}$. A similar approach can be used for computing a lower bound on the DL capacity, as done in [7,8].
} 
(MRC), and it is the signal processing technique used throughout this thesis. The idea behind MRC is to maximize the desired signal (the first term in (24)) and it is a good choice for cases with low signal-to-noise ratio (SNR). In addition, it has linear computational complexity in $M$ and $K$, and it can be implemented with a distributed architecture. There are other choices for the received combining matrix, for instance, zero forcing (ZF) and multicell MMSE (mMMSE) which are defined as

$$
\mathbf{A}= \begin{cases}\hat{\mathbf{H}}_{00}\left(\hat{\mathbf{H}}_{00}^{H} \hat{\mathbf{H}}_{00}\right)^{-1} & \mathrm{ZF} \\ \left(\sum_{l \in \Phi} \sum_{i=1}^{K} \rho_{l i}^{\mathrm{RP}}\left(\hat{\mathbf{h}}_{0 l i} \hat{\mathbf{h}}_{0 l i}^{H}+\left(1-\gamma_{l i}\right) \beta_{0 l i} \mathbf{I}_{M}\right)+\sigma^{2} \mathbf{I}_{M}\right)^{-1} \hat{\mathbf{H}}_{00} \mathbf{D}_{\rho_{0}^{\mathrm{RP}}} & \text { mMMSE }\end{cases}
$$

where $\hat{\mathbf{H}}_{00}=\left[\hat{\mathbf{h}}_{001}, \ldots, \hat{\mathbf{h}}_{00 K}\right]$ and $\mathbf{D}_{\rho_{0}^{\mathrm{RP}}}=\operatorname{diag}\left(\rho_{01}^{\mathrm{RP}}, \ldots, \rho_{0 K}^{\mathrm{RP}}\right)$. With ZF the idea is to cancel the intracell interference (see the second term in (24)) that is introduced by other users in the same cell as $\mathrm{UE}_{0 k}$. This is a good choice in cases with high SNR where the interference effect is strong. The mMMSE is the optimal linear processing where a balance between interference suppression and desired signal amplification is achieved taking into account the effects of intercell interference.

Note that ZF and mMMSE perform better than MRC in high SNR scenarios, however they also have higher computational complexity due to the matrix inversion needed (see (29)). In multicell scenarios where the channels are i.i.d. Rayleigh fading and low SNR is considered, the gap between mMMSE and MRC becomes small as $M$ increases (see Section 2.5). It is worth mentioning that, in scenarios where the channel estimates are nonGaussian (as the ones studied in this thesis) the analysis with $\mathrm{ZF}$ and mMMSE tends to be analytically intractable. Therefore, in this thesis, the analysis is focused on MRC where closed-form expressions are computed and clear insights into the phenomena that affect the performance can be obtained.

\subsection{Performance of Massive MIMO}

To evaluate the performance of Massive MIMO, the following three main performance metrics are used throughout this thesis: SE, power consumption and EE. The SE is obtained from the capacity analysis depicted in Section 2.4. The general power consumption model used in this thesis is based on [45], such that the power consumption per cell is

$$
\mathrm{P}_{\mathrm{CELL}}=\mathrm{P}_{\mathrm{TX}-\mathrm{BS}}+\mathrm{P}_{\mathrm{FIX}}+K \mathrm{P}_{\mathrm{UE}}+M \mathrm{P}_{\mathrm{RF}}+\mathrm{P}_{\mathrm{CE}}+\mathrm{P}_{\mathrm{LP}}+\mathrm{P}_{\mathrm{CD}}\left(B_{\mathrm{W}} \mathrm{SE}_{\mathrm{CELL}}\right)
$$


where $\mathrm{P}_{\mathrm{TX}-\mathrm{BS}}$ accounts for the transmission power per BS, $\mathrm{P}_{\mathrm{FIX}}$ is the fixed power consumption (e.g., site cooling), $\mathrm{P}_{\mathrm{UE}}$ is the power consumption per $\mathrm{UE}, \mathrm{P}_{\mathrm{RF}}$ is the power consumption per RF chain at the BS, $\mathrm{P}_{\mathrm{CE}}$ is the power consumed by channel estimation processing, $\mathrm{P}_{\mathrm{LP}}$ is the power spent on linear signal processing, and $\mathrm{P}_{\mathrm{CD}}$ is the power consumption for coding and decoding tasks that depends on the data rate per cell. The SE per cell is defined as $\mathrm{SE}_{\mathrm{CELL}}$ so that the data rate per cell is given by $B_{\mathrm{W}} \mathrm{SE}_{\mathrm{CELL}}[\mathrm{bit} / \mathrm{s}]$ when sampling at the Nyquist rate. Finally, the EE is defined as

$$
\mathrm{EE}=\frac{B_{\mathrm{W}} \mathrm{SE}_{\mathrm{CELL}}}{\mathrm{P}_{\mathrm{CELL}}}
$$

The model in (30) includes the main sources of power consumption, and depending on the focus of the analysis, some terms might be lumped together into fixed terms or further expanded by using more detailed models. In particular, when studying the impact of the ADC bit resolution on the power consumption, the term $\mathrm{P}_{\mathrm{RF}}$ is further expanded as

$$
\mathrm{P}_{\mathrm{RF}}=\mathrm{D}_{0}+2 \mathrm{P}_{\mathrm{ADC}}
$$

where $\mathrm{D}_{0}$ includes the power consumption per $\mathrm{RF}$ chain that is independent of the ADC bit resolution and

$$
\mathrm{P}_{\mathrm{ADC}}=\mathrm{D}_{1} 2^{\varphi b}
$$

is the power consumed by the ADC. The term $b$ corresponds to the $\mathrm{ADC}$ bit resolution and $\mathrm{D}_{1} \geq 0$ and $\varphi \geq 0$ are set constants. Typical values are $\mathrm{D}_{1} \in\left[10^{-8}, 10^{-7}\right]\left[\mathrm{W} /\right.$ conversion step], $\varphi \in[1,2]{ }^{4}$ The model in (33) is chosen to encompass different architectures and implementations of ADCs found in the literature [33,46-48].

When varying the ADC bit resolution, the power consumption of channel estimation and signal processing tasks is also affected since it depends on the number of bits used for baseband operations. By counting the number of linear algebra multiplication operations, the power consumption of channel estimation and signal processing can be approximated by

$$
\mathrm{P}_{\mathrm{CE}}+\mathrm{P}_{\mathrm{LP}}=\frac{B_{\mathrm{W}}}{L} 2 M K b
$$

where $L$ is the computational efficiency of the hardware at the BS, measured in [bit operations/Joule].

\footnotetext{
${ }^{4}$ The choice of $\mathrm{D}_{1}$ and $\varphi$ can be related to the figure-of-merit (FoM) of ADCs where small values of $D_{1}$ are paired with larger values of $\varphi$ and vice versa [46].
} 
To illustrate the key benefits and characteristics of Massive MIMO, consider the multicell MU-MIMO model presented in the previous sections under MRC. The SE of $\mathrm{UE}_{0 k}$ base on the lower bound on the capacity in (26) is given by $[7,8]$

$$
\mathrm{SE}_{0 k}=\left(\frac{\tau_{u l}-\tau_{p}}{\tau_{c}}\right) \log _{2}\left(1+\frac{M \beta_{00 k} \rho_{0 k}^{\mathrm{RP}}}{\sum_{(l, i) \in \mathcal{P}_{0 k} \backslash(0, k)} \frac{\beta_{0 i}^{2} q_{l i}^{\mathrm{RP}} \rho_{l i}^{\mathrm{RP}}}{\beta_{00 k} q_{0 k}^{\mathrm{RP}}}+\frac{1}{\gamma_{0 k}}\left(\sum_{l \in \Phi} \sum_{i=1}^{K} \beta_{0 l i} \rho_{l i}^{\mathrm{RP}}+\sigma^{2}\right)}\right)
$$

where the numerator of the SINR is referred to as the coherent gain and it scales with $M$, the first term in the denominator is called the coherent interference and the second term is the non-coherent interference. In this thesis, coherent interference refers to the interference that combines constructively in the detection process, because it comes from the same spatial direction as the channel estimates, and therefore scales with $M$. Instead, non-coherent interference refers to interference that combines in a non-constructive way and does not scale with $M$.

The closed form expression in (35) allows for a simple evaluation of the SE and shows key insights into the performance of Massive MIMO. In what follows, some numerical results are also introduced to exemplify some of the main properties of Massive MIMO. Three cases of study for the numerical results are introduced which correspond to Figures 4-6. To deliver the main insights in a concise manner, first the main assumptions on each case of study are introduced, and second, a point-by-point explanation of the key characteristics of Massive MIMO is presented with references to previous analysis and said numerical results.

Case 1) Multicell MU-MIMO system: Figure 4 shows the SE per cell for a multicell MU-MIMO system with squared cells of side length $R_{c}[\mathrm{~m}]$ where $K$ UEs are distributed uniformly within each cell with a minimum distance to the serving BS of $d_{\min }=10$ [m]. The LSF coefficient is given by $\beta_{0 l i}=\omega^{-1} d_{0 l i}^{-\alpha}$ where $d_{0 l i}$ is the distance between $\mathrm{UE}_{l i}$ and $\mathrm{BS}_{0}$ (measured in meters), $\alpha$ is the pathloss exponent, and $\omega$ is the pathloss at a reference distance of 1 meter. This LSF model is used for all three numerical study cases. The wrap-around technique is used to avoid edge effects and underestimate the interference level. Statistical channel inversion power control is used for both the pilot and data symbols, such that, $q_{l i}^{\mathrm{RP}}=\rho_{l i}^{\mathrm{RP}}=\rho / \beta_{0 l i}$ where $\rho$ is a parameter used to set the SNR level. This assumption is also used for all three numerical study cases. Table 1 contains the main parameters used for the numerical study. 
Table 1: Parameters for numerical analysis.

\begin{tabular}{c|c|c} 
Parameter & Symbol & Value \\
\hline Fixed propagation loss at $1[\mathrm{~m}]$ & $\omega$ & $35[\mathrm{~dB}]$ \\
Cell radius & $R_{c}$ & $200[\mathrm{~m}]$ \\
Pathloss exponent & $\alpha$ & 3.76 \\
System bandwidth & $\mathrm{B}_{\mathrm{W}}$ & $20[\mathrm{MHz}]$ \\
Const. related to ADC saturation level & $\zeta$ & 1.6 \\
Consts. related to the ADC FoM & $\mathrm{D}_{1}$ & $10^{-7}[\mathrm{~W} /$ conv-step $]$ \\
& $\varphi$ & 1 \\
Fixed power consumption & $\mathrm{P}_{\mathrm{FIX}}$ & $10[\mathrm{~W}]$ \\
Circuit power per active UE & $\mathrm{P}_{\mathrm{UE}}$ & $0.1[\mathrm{~W}]$ \\
Circuit power per BS antenna (ind. of $b)$ & $\mathrm{D}_{0}$ & $0.1[\mathrm{~W}]$ \\
Power coding/decoding & $\mathrm{P}_{\mathrm{CD}} B_{\mathrm{W}}$ & $0.023[\mathrm{~W} / \mathrm{bit}]$ \\
Computational efficiency & $L / B_{\mathrm{W}}$ & $20480[\mathrm{bit}$ op./W] \\
Noise power & $\sigma^{2} B_{\mathrm{W}}$ & $2 \cdot 10^{-13}[\mathrm{~W}]$ \\
\hline
\end{tabular}

Case 2) Quantized SIMO system. In Figure 5, the normalized distortion power for a quantized SIMO system with $M$ antennas at the receiver is illustrated. Here, the received signal for all samples in the coherence block is given by

$$
\mathbf{Y}=\mathbb{Q}\left(\mathbf{h} \mathbf{x}^{T}+\mathbf{N}\right) \in \mathbb{C}^{M \times \tau_{c}}
$$

where $\mathbf{x} \in \mathbb{C}^{\tau_{c} \times 1}$ is the transmitted signal that is composed of $\tau_{p}=1$ pilot symbol and the rest correspond to data symbols. The noise is given by $\mathbf{N}$ with elements distributed as $[\mathbf{N}]_{m j} \sim \mathcal{C N}\left(0, \sigma^{2}\right)$. The operator $\mathbb{Q}(\cdot)$ stands for quantization with optimized levels as in [49]. The channel estimate $\hat{\mathbf{h}}$ is computed based on LMMSE estimation following the same approach as in Section 2.3 with observation vector given by the quantized vector taken from the $\tau_{p}$ column of $\mathbf{Y}$ in (36). The data estimate for an arbitrary symbol in the coherence block, denoted with the index $j$, is computed as $\hat{s}_{j}=\left(\frac{\hat{\mathbf{h}}}{\|\hat{\mathbf{h}}\|}\right)^{H}[\mathbf{Y}]_{j}$ and the normalized distortion is defined as

$$
n_{d}=\frac{\mathbb{E}\left\{\left|\hat{s}_{j}-\mathbb{E}\left\{\hat{s}_{j} s_{j}^{*}\right\} s_{j}\right|^{2}\right\}}{\sigma^{2} M}
$$

where the normalization is done with respect to the number of antennas at the receiver and the noise energy per symbols. 


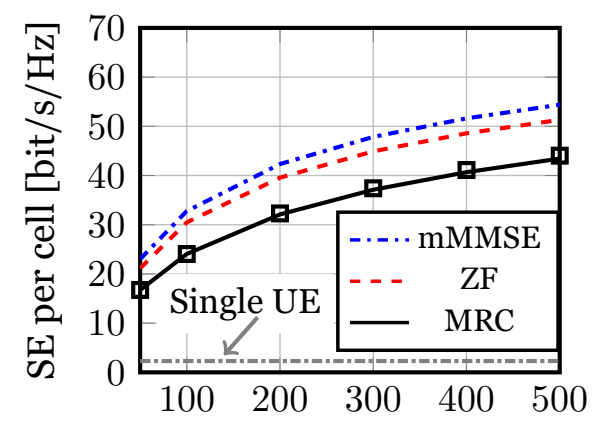

Number of BS antennas $M$

(a) Low SNR, $\frac{\rho}{\sigma^{2}}=-6[\mathrm{~dB}]$.

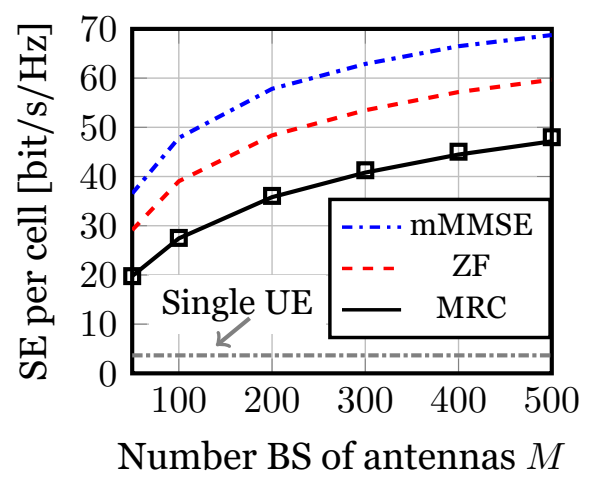

(b) High SNR, $\frac{\rho}{\sigma^{2}}=10[\mathrm{~dB}]$.

Figure 4: SE per cell versus $M$ in a multicell scenario for $K=10$ and $\tau_{p}=10$. The lines correspond to (28) and the squared marks follow from (35).

Case 3) Quantized single-cell MU-MIMO system. Figure 6 shows the $\mathrm{EE}$ of a single cell Massive MIMO system with imperfect CSI where the BS deploys $M$ antennas and serves $K$ UEs. The quantization is implemented with optimized levels as in [49] and the received signal at the BS is

$$
\mathbf{Y}=\mathbb{Q}\left(\sum_{i=1}^{K} \mathbf{h}_{i} \sqrt{p_{i}} \mathbf{x}_{i}^{T}+\mathbf{N}\right) \in \mathbb{C}^{M \times \tau_{c}}
$$

where $\mathbf{x}_{i}$ encompass the transmitted symbols from $\mathrm{UE}_{i}$ with energy per symbol (for both the pilot and data) $p_{i}$, and $\mathbf{h}_{i} \sim \mathcal{C N}\left(\mathbf{0}, \mathbf{I}_{M} \beta_{i}\right)$ is the channel between $\mathrm{UE}_{i}$ and the BS. LMMSE channel estimation is used following the same approach as in Section 2.3 and the SE is computed based on the capacity lower bound in (26) and (27). The power consumption is given by (30), (32), (33), and (34). Table 1 depicts the values of the parameters used for the numerical results.

In what follows, the main characteristics of Massive MIMO are explained based on the results in Section 2.4, (31), (35), and Figures 4-6.

Spatial multiplexing. The SE in (35) corresponds to $\mathrm{UE}_{0 k}$ and $K$ parallel data streams are transmitted simultaneously in each cell. Thus, the sum SE in cell 0 is $\sum_{i=1}^{K} \mathrm{R}_{0 i}$. By assuming a power control scheme for UEs in cell 0 that provides uniform rate, the sum rate in cell 0 is then $\sum_{i=1}^{K} \mathrm{R}_{0 i}=K \mathrm{R}_{0}$ which grows linearly with $K$. In Figure 4, the effect of spatial multiplexing is exemplified by comparing the result for serving 


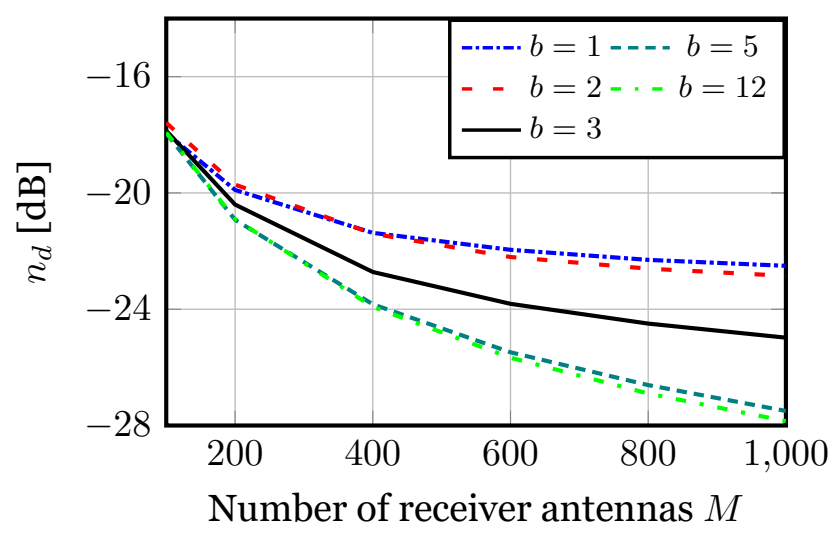

Figure 5: Normalized distortion power versus $M$ for quantized SIMO system with $\frac{\rho}{\sigma^{2}}=0[\mathrm{~dB}]$.

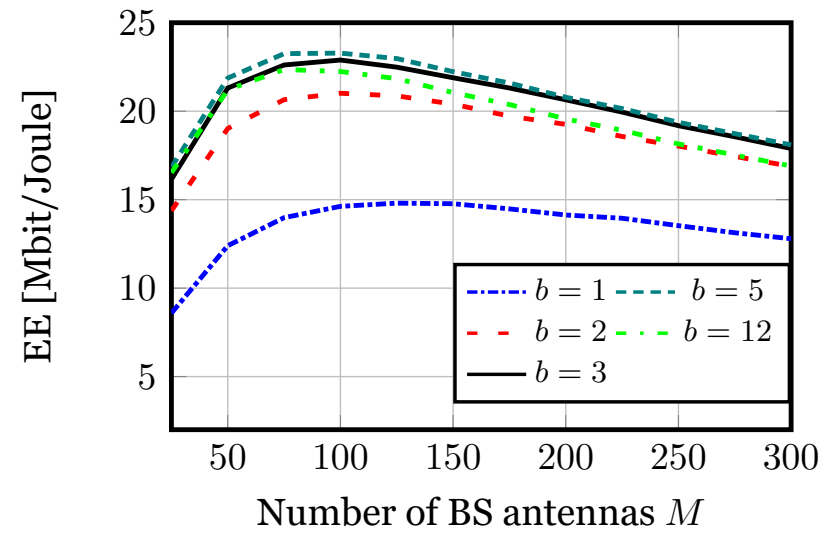

Figure 6: EE versus $M$ with $K=10$ and $\frac{\rho}{\sigma^{2}}=-6[\mathrm{~dB}]$ for quantized single-cell Massive MIMO.

a single UE per cell with $M=50$ (gray bottom line) and the rest of the curves.

Coherent processing gain. The numerator of the SINR inside the logarithm in (35) scales linearly with the number of BS antennas and the $\mathrm{SE}$ is an increasing function of $M$. In a single cell scenario $\mathcal{P}_{0 k}=(0, k)$, which means that the SINR scales linearly with $M$ and the SE scales logarithmically with $M$. This result is exemplified in Figure 4 for a multicell scenario where it is shown that the SE in an increasing function of $M$. 
Pilot contamination. This phenomenon causes three key effects: $i$ ) It decreases the estimation quality by reducing $\gamma_{0 k}$. ii) It decreases the coherent gain as consequence of having lower $\gamma_{0 k}$ (see the second term in the denominator of the SINR in (35)). iii) It causes coherent interference that reduces the SE. For i.i.d. Rayleigh fading this effect can be seen in the saturation of the SE, in Figure 4, as $M$ grows large.

Transmission power reduction. Scaling down the transmission data power as $\rho_{l i}^{\mathrm{RP}}=\rho_{l i} / \sqrt{M}$, results in an SINR that is still an increasing function of $M$ (see (35)). In the case of a single cell scenario, the SE scales logarithmically with $\sqrt{M}$.

Linear processing. Thanks to asymptotic favorable propagation and channel hardening, Massive MIMO with linear processing is able to provide high SE per cell, as illustrated in Figure 4. In particular, notice that in the low SNR regime (see Figure 4a) the SE with MRC is close to the SE with $\mathrm{ZF}$ and mMMSE. Whereas, in the high SNR case the benefits of $\mathrm{ZF}$ and $\mathrm{mMMSE}$ are more pronounced.

Low end hardware. The coherent processing gain and excess number of BS antennas, increase the tolerance of non-coherent distortions from low end hardware. In the case of quantization, this result is exemplified in Figure 5 where the distortion power normalized by $M$ decreases as $M$ grows large. Notice that the power consumption of ADCs increases exponentially with the ADC bit resolution (see (33)), thus Massive MIMO is also able to reduce circuit power by relaxing the ADC bit resolution. As a results, high EE can by achieved by Massive MIMO which is illustrated in Figure 6. 


\section{Chapter 3}

\section{Alternative Massive MIMO Designs}

This chapter introduces the alternative designs for Massive MIMO studied in this thesis, and the main assumptions and insights from the analysis are explained.

\subsection{Superimposed Pilots}

The main idea behind SP in Massive MIMO is to eliminate the pilot overhead and reduce the pilot contamination effect to offer a higher SE than with RP. To study SP, a TDD transmission protocol is assumed, as depicted in Figure 7, where the pilot and data symbols are superimposed in the UL. Channel estimates are constructed from the received signal in the UL and, in turn, are used for UL and DL signal processing via channel reciprocity.

A multicell MU-MIMO system model is used, similar to the one in Section 2.3, where each BS deploys $M$ antennas and $K$ UEs are served in each cell. A set $\mathcal{U}_{\tau_{u l}}$ of mutually orthogonal pilot sequences with cardinality $\tau_{u l}$ is available for channel estimation. The pilot used by $\mathrm{UE}_{0 k}$ is $\varphi_{0 k} \in \mathcal{U}_{\tau_{u l}}$ with elements having unit modulus, that is, $\left|\left[\boldsymbol{\varphi}_{0 k}\right]_{j}\right|=1 \forall j \in\left\{1, \ldots, \tau_{u l}\right\}$. Different pilots are assigned within each individual cell, which means that they are mutually orthogonal, that is

$$
\boldsymbol{\varphi}_{0 k}^{H} \boldsymbol{\varphi}_{0 i}= \begin{cases}\tau_{u l} & \text { for } k=i \\ 0 & \text { for } k \neq i\end{cases}
$$

In the case of SP, there are more pilot sequences available than with RP, however the number of pilots is still limited by the size of the coherence block 


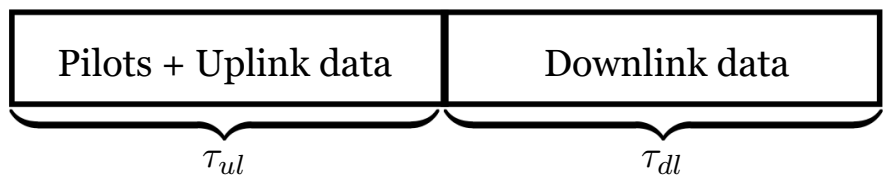

Figure 7: TDD transmission protocol with SP.

and therefore, in dense scenarios with many BSs and UEs, pilots would still need to be reused between cells. The set $\mathcal{S}_{0 k}$ encloses the indices of all UEs that use the same pilot as $\mathrm{UE}_{0 k}$, including itself, and is defined as

$$
\mathcal{S}_{0 k}=\left\{(l, i): \varphi_{0 k}^{H} \boldsymbol{\varphi}_{l i} \neq 0\right\} .
$$

The received signal in the $\mathrm{UL}$ at $\mathrm{BS}_{0}$ is

$$
\mathbf{Z}_{0}=\sum_{l \in \Phi} \sum_{i=1}^{K} \mathbf{h}_{0 l i}\left(\sqrt{q_{l i}^{\mathrm{SP}}} \boldsymbol{\varphi}_{l i}^{T}+\sqrt{\rho_{l i}^{\mathrm{SP}}} \mathbf{s}_{l i}^{T}\right)+\mathbf{N}_{0} \in \mathbb{C}^{M \times \tau_{u l}}
$$

where $\mathbf{s}_{l i} \in \mathbb{C}^{\tau_{u l} \times 1}$ is the vector composed of the UL data symbols transmitted by $\mathrm{UE}_{l i}$. The noise matrix is $\mathbf{N}_{0}=\left[\mathbf{n}_{01}, \ldots, \mathbf{n}_{0 \tau_{u l}}\right]$ with $\mathbf{n}_{0 j} \sim \mathcal{C N}\left(\mathbf{0}, \mathbf{I}_{M} \sigma^{2}\right)$ and $\sigma^{2}$ is the average noise energy per symbol. The transmission energy per pilot symbol from $\mathrm{UE}_{l i}$ is $q_{l i}^{\mathrm{SP}}$, and the average transmission energy per data symbol is $\rho_{l i}^{\mathrm{SP}}$. Note that, with SP the pilot and data symbols are transmitted simultaneously. Thus, the transmission power needs to be shared between pilot and data symbols, so that $q_{l i}^{\mathrm{SP}}+\rho_{l i}^{\mathrm{SP}}$ is the total average transmission energy per symbol from $\mathrm{UE}_{l i}$. LMMSE channel estimation is used where the observations are given by performing a de-spreading operation on $\mathbf{Z}_{0}$ such that

$$
\begin{aligned}
& \mathbf{z}_{0 k}= \mathbf{Z}_{0} \boldsymbol{\varphi}_{l i}^{*} / \sqrt{\tau_{u l}} \\
&=\sum_{(l, i) \in \mathcal{S}_{0 k}} \mathbf{h}_{0 l i} \sqrt{q_{l i}^{\mathrm{SP}} \tau_{u l}}+\underbrace{\sum_{l \in \Phi} \sum_{i=1}^{K} \mathbf{h}_{0 l i} \sqrt{\frac{\rho_{l i}^{\mathrm{SP}}}{\tau_{u l}}} \mathbf{s}_{l i}^{T} \boldsymbol{\varphi}_{l i}^{*}}_{\begin{array}{c}
\text { Interference in channel } \\
\text { estimates due to data symbols }
\end{array}}+\mathbf{N}_{0} \frac{\boldsymbol{\varphi}_{l i}^{*}}{\sqrt{\tau_{u l}}} \in \mathbb{C}^{M \times 1} .
\end{aligned}
$$

The following lemma summarizes the results for obtaining the LMMSE channel estimates of $\mathbf{h}_{00 k}$. 
Lemma 2. The LMMSE estimate of $\mathbf{h}_{00 k}$ with SP based on $\mathbf{z}_{0 k}$ is

$$
\hat{\mathbf{h}}_{00 k}=\frac{\gamma_{0 k}}{\sqrt{q_{0 k}^{\mathrm{SP}} \tau_{u l}}} \mathbf{z}_{0 k}
$$

where

$$
\gamma_{0 k}=\frac{q_{0 k}^{\mathrm{SP}} \tau_{u l} \beta_{00 k}}{\sum_{(l, i) \in \mathcal{S}_{0 k}} q_{l i}^{\mathrm{SP}} \tau_{u l} \beta_{0 l i}+\sum_{l \in \Phi} \sum_{i=1}^{K} \rho_{l i}^{\mathrm{SP}} \beta_{0 l i}+\sigma^{2}} .
$$

The covariance of the channel estimate is

$$
\mathbb{E}\left\{\hat{\mathbf{h}}_{00 k} \hat{\mathbf{h}}_{00 k}^{H}\right\}=\gamma_{0 k} \beta_{00 k} \mathbf{I}_{M}
$$

Proof. It follows from computing the linear channel estimate of $\mathbf{h}_{00 k}$ that minimizes the MSE, based on the observations $\mathbf{z}_{0 k}$ in (42). This result is based on applying standard LMMSE estimation techniques [43, Ch. 15.8] to the problem at hand.

To gain insights into the SE performance comparison between RP and SP, understanding the differences between the channel estimates with RP and SP is essential. These differences are summarized as follows:

\section{SP has more de-spreading gain.}

Since with SP the whole UL part of the coherence block is used for both pilots and data, the number of observations available for channel estimation is higher than with RP, that is, $\tau_{u l} \geq \tau_{p}$. This means that the numerator in (44) with SP, referred to as the de-spreading gain, is higher than the numerator in (20) with RP.

\section{Channel estimates with SP have less interference from pilot con- tamination.}

The effect of pilot contamination in the channel estimates depends on the UEs belonging to the set $\mathcal{S}_{0 k}$, as shown in the first term in the denominator of (44) with SP, or to the set $\mathcal{P}_{0 k}$, in the first term in the denominator of (20) with RP. Since the number of available pilots is higher with SP than with RP, the pilots can be reused less frequently in the network. Thus, the cardinality of the set $\mathcal{S}_{0 k}$ is lower than the one of the set $\mathcal{P}_{0 k}$. In addition, the pathloss between $\mathrm{BS}_{0}$ and the UEs in the set $\mathcal{S}_{0 k}$ is higher than for UEs in the set $\mathcal{P}_{0 k}$. As a result, the effect of the pilot contamination is reduced with SP compared to RP. 


\section{Channel estimates with SP have interference from data symbols that reduces the estimation quality.}

As shown in the second term in (42), the observations with SP (after de-spreading) have an extra amount of interference introduced by the data symbols, which does not exist with RP. This interference reduces the quality of the channel estimates which appears as the second term in the denominator of (44). Notice also that this interference comes from all UEs in the system, including the UE of interest itself. It is worth to stress that the presence of this data interference in (42) changes the distribution of the observation vector $\mathbf{z}_{0 k}$ which is no longer Gaussian, but depends on the product of the channel and data symbols. This makes the analytical derivation of the MMSE estimator in closed-form less tractable and valuable insights tend to be lost in complicated expressions. For this reason in this thesis, LMMSE channel estimation is used instead. This means that the LMMSE channel estimates with SP are not coinciding with the MMSE channel estimates as it does in the case with RP. In addition, the channel estimates with SP and their corresponding estimation errors are uncorrelated but not statistically independent. This fact calls for a different analysis from the one in $[7,8]$, when computing closed-form expressions for the effective SINR in (28).

\section{The interference from data creates correlation between channel estimates and data symbols.}

The observations $\mathbf{z}_{0 k}$ depend on the data signals through the second term in (42), and thus, the channel estimates and data symbols are correlated. This means that when the channel estimates are used for linear signal processing to obtain the data estimates, extra interference terms appear which are caused by the correlation between data symbols and channel estimates. This phenomena is similar to pilot contamination, but caused in a different way.

The detection is based on maximum ratio linear processing due to its low complexity, good performance in low SNR and multicell scenarios (see Section 2.5), and analytical tractability with non-Gaussian channel estimates. The SE expressions are based on the analysis given in Section 2.4. Notice that with SP, the UL signal for both the pilot estimation and data detection is the same, see (41). Since the pilot symbols are known at the BS, they can be subtracted from the received signal by using the knowledge from the channel estimates, which may be useful to reduce interference. This effect is studied in two cases: $i$ ) The channel estimates are used to subtract the estimated versions of the received pilot symbols such that the received signal 
for data detection processing is

$$
\mathbf{Z}_{0}=\sum_{l \in \Phi} \sum_{i=1}^{K}\left(\left(\mathbf{h}_{0 l i}-\hat{\mathbf{h}}_{0 l i}\right) \sqrt{q_{l i}^{\mathrm{sP}}} \boldsymbol{\varphi}_{l i}^{T}+\mathbf{h}_{0 l i} \sqrt{\rho_{l i}^{\mathrm{SP}}} \mathbf{s}_{l i}^{T}\right)+\mathbf{N}_{0} \in \mathbb{C}^{M \times \tau_{u l}} .
$$

ii) The received pilot symbols are perfectly removed from the received signal for the data detection process, that is,

$$
\mathbf{Z}_{0}=\sum_{l \in \Phi} \sum_{i=1}^{K} \mathbf{h}_{0 l i} \sqrt{\rho_{l i}^{\mathrm{sP}}} \mathbf{s}_{l i}^{T}+\mathbf{N}_{0} \in \mathbb{C}^{M \times \tau_{u l}} .
$$

The first case in (46) corresponds to a more practical approach to see what are the gains from subtracting the pilots. However, the analytical expressions become involved and little insights can be obtained from them, thus only numerical results are provided for this case. In the second case, the received pilot symbols are perfectly removed in order to obtain an upper bound on the performance that can be achieved with SP. In what follows, the signal processing with SP is referred to as: "no pilot subtraction" when (41) is taken as the received data signal, "estimated pilot subtraction" when (46) is used, and "perfect pilot subtraction" when using (47).

The assignment of pilot sequences among cells affects the pilot contamination and makes it difficult to obtain a clear comparison between the performance of RP and SP. Thus, a random pilot allocation strategy is introduced to obtain clear insights into the effect of the pilot length on the pilot contamination with both SP and RP. This is implemented by having each BS selecting $K$ mutually orthogonal pilots at random and assigning them to the UEs in its cell. This means that

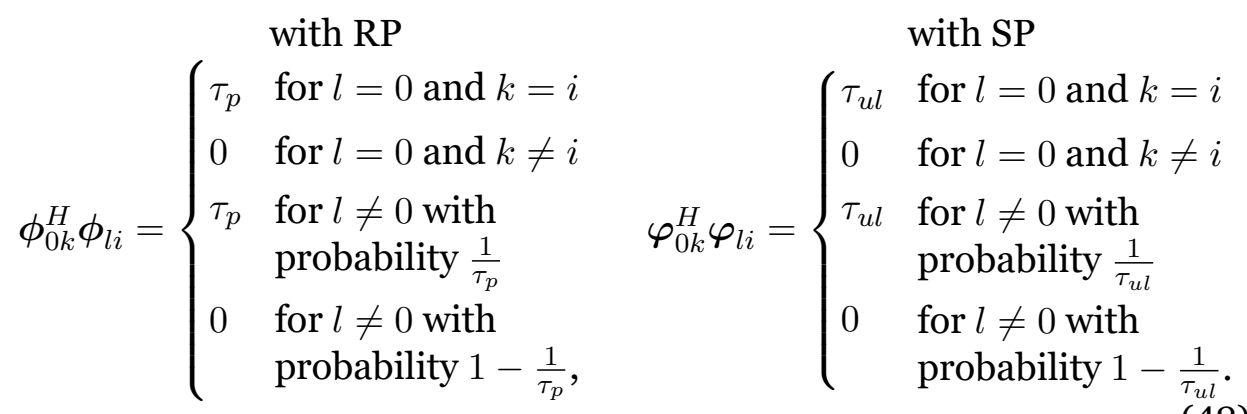

The probability that in a given cell $l \neq 0$ there is a UE with the same pilot as $\mathrm{UE}_{0 k}$ is $K / \tau_{p}$ with RP, and $K / \tau_{u l}$ with SP. In the case where in said cell $l \neq 0$ there is such a UE, the individual selection of this UE is done uniformly at 
random. Based on these assumptions, the following results hold

$$
\begin{gathered}
\mathbb{E}\left\{\sum_{(l, i) \in \mathcal{P}_{0 k} \backslash(0, k)}^{K} q_{l i}^{\mathrm{RP}} \beta_{0 l i}\right\}=\mathbb{E}\left\{\sum_{l \in \Phi \backslash 0} \sum_{i=1}^{K} \frac{\phi_{l i}^{H} \boldsymbol{\phi}_{0 k}}{\tau_{p}} q_{l i}^{\mathrm{RP}} \beta_{0 l i}\right\}=\sum_{l \in \Phi \backslash 0} \frac{K}{\tau_{p}} \frac{1}{K}\left(\sum_{i=1}^{K} q_{l i}^{\mathrm{RP}} \beta_{0 l i}\right), \\
\mathbb{E}\left\{\sum_{(l, i) \in \mathcal{S}_{0 k} \backslash(0, k)}^{K} q_{l i}^{\mathrm{SP}} \beta_{0 l i}\right\}=\mathbb{E}\left\{\sum_{l \in \Phi \backslash 0} \sum_{i=1}^{K} \frac{\boldsymbol{\varphi}_{l i}^{H} \boldsymbol{\varphi}_{0 k}}{\tau_{u l}} q_{l i}^{\mathrm{SP}} \beta_{0 l i}\right\}=\sum_{l \in \Phi \backslash 0} \frac{K}{\tau_{u l}} \frac{1}{K}\left(\sum_{i=1}^{K} q_{l i}^{\mathrm{SP}} \beta_{0 l i}\right) .
\end{gathered}
$$

Thus, indicators of the channel estimation quality under random pilot allocation with RP, and SP, are defined as

$$
\begin{aligned}
& \gamma_{0 k}^{\mathrm{RP}}=\mathbb{E}\left\{\left.\frac{1}{\gamma_{0 k}}\right|_{\text {eq. (20) }}\right\}^{-1}=\frac{q_{0 k}^{\mathrm{RP}} \tau_{p} \beta_{00 k}}{q_{0 k}^{\mathrm{RP}} \tau_{p} \beta_{00 k}+\sum_{l \in \Phi \backslash 0} \sum_{i=1}^{K} q_{l i}^{\mathrm{RP}} \beta_{0 l i}+\sigma^{2}}, \\
& \gamma_{0 k}^{\mathrm{SP}}=\mathbb{E}\left\{\left.\frac{1}{\gamma_{0 k}}\right|_{\text {eq. (44) }}\right\}^{-1}=\frac{q_{0 k}^{\mathrm{SP}} \tau_{u l} \beta_{00 k}}{q_{0 k}^{\mathrm{SP}} \tau_{u l} \beta_{00 k}+\sum_{l \in \Phi \backslash 0} \sum_{i=1}^{K} q_{l i}^{\mathrm{SP}} \beta_{0 l i}+\sum_{l \in \Phi} \sum_{i=1}^{K} \rho_{l i}^{\mathrm{SP}} \beta_{0 l i}+\sigma^{2}},
\end{aligned}
$$

where a clear dependency on $\tau_{p}$ and $\tau_{u l}$ can be identified.

In what follows, the main analytical and numerical results from Papers $A$ and $B$ are presented, and the main insights are explained. The analytical results are summarized in the following lemmas and theorems corresponding to the SE expressions obtained for the UL and DL with RP, and with SP, respectively. ${ }^{1}$

\section{ULSE with RP:}

Lemma 3. A lower bound on the UL capacity of $\mathrm{UE}_{0 k}$ with $R P$ and $M R C$ detection is

$$
\mathrm{R}_{0 k}^{\mathrm{UL}-\mathrm{RP}}=\left(\frac{\tau_{u l}-\tau_{p}}{\tau_{c}}\right) \log _{2}\left(1+\mathrm{SINR}_{0 k}^{\mathrm{UL}-\mathrm{RP}}\right)
$$

\footnotetext{
${ }^{1}$ Note that the novel expressions correspond to the case with SP. However, the expressions with RP are added for comparison purposes.
} 
where $\mathrm{SINR}_{0 k}^{\mathrm{UL}-\mathrm{RP}}$ is the effective SINR of $\mathrm{UE}_{0 k}$ given by

$$
\operatorname{SINR}_{0 k}^{\mathrm{UL}-\mathrm{RP}}=\underbrace{\frac{M \rho_{0 k}^{\mathrm{RP}} \beta_{00 k}}{\tau_{p}} \sum_{l \in \Phi \backslash 0} \sum_{i=1}^{K} \frac{\rho_{l i}^{\mathrm{RP}} q_{l i}^{\mathrm{RP}}}{q_{0 k}^{\mathrm{RP}}} \frac{\beta_{0 l i}^{2}}{\beta_{00 k}}}_{\begin{array}{c}
\text { Coherent interference caused } \\
\text { by pilot contamination }
\end{array}}+\underbrace{\frac{1}{\gamma_{0 k}^{\mathrm{RP}}}\left(\sum_{l \in \Phi} \sum_{i=1}^{K} \rho_{l i}^{\mathrm{RP}} \beta_{0 l i}+\sigma^{2}\right)}_{\begin{array}{c}
\text { Non-coherent interference } \\
\text { plus noise }
\end{array}} .
$$

Proof. It follows from the analysis shown in Section 2.4 and can be found in $[7,8]$. The proof of the closed-form expression in (54) considering the effect of random pilot allocation follows from (49) and is given in Paper A.

\section{UL SE with SP:}

Theorem 1. A lower bound on the UL capacity of $\mathrm{UE}_{0 k}$ with SP, MRC detection, and no pilot subtraction is

$$
\mathrm{R}_{0 k}^{\mathrm{UL}-\mathrm{SP}}=\frac{\tau_{u l}}{\tau_{c}} \log _{2}(1+\underbrace{\frac{M \rho_{0 k}^{\mathrm{SP}} \beta_{00 k}}{I_{\mathrm{C}-\mathrm{I}}^{\mathrm{LP}}+I_{\mathrm{NC}-\mathrm{IP}}^{\mathrm{UL}}}}_{\mathrm{SINR}_{0 k}^{\mathrm{UL}-\mathrm{SP}}})
$$

where $\mathrm{SINR}_{0 k}^{\mathrm{UL}-\mathrm{SP}}$ is the effective SINR of $\mathrm{UE}_{0 k}$. The term $I_{\mathrm{C}-\mathrm{I}}^{\mathrm{UL}} \mathrm{SP}$ corresponds to the coherent interference and $I_{\mathrm{NC}-\mathrm{I}}^{\mathrm{UL}-\mathrm{SP}}$ is the non-coherent interference plus noise, which are given by

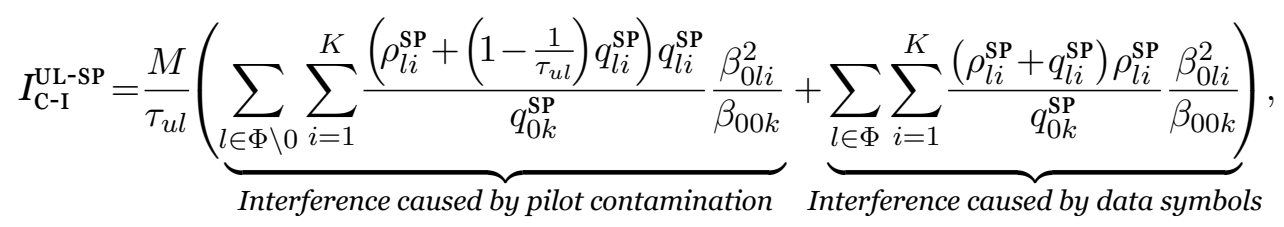

$$
\begin{aligned}
I_{\mathrm{NC}-\mathrm{I}}^{\mathrm{UL}-\mathrm{SP}}= & \frac{2}{\tau_{u l}} \rho_{0 k}^{\mathrm{SP}} \beta_{00 k}+\frac{2}{\tau_{u l}^{2}} \sum_{l \in \Phi \backslash 0} \sum_{i=1}^{K} \frac{q_{l i}^{\mathrm{SP}} \rho_{l i}^{\mathrm{SP}}}{q_{0 k}^{\mathrm{SP}}} \frac{\beta_{0 l i}^{2}}{\beta_{00 k}}+\frac{1}{\tau_{u l}^{2}} \sum_{l \in \Phi} \sum_{i=1}^{K} \frac{\left(\rho_{l i}^{\mathrm{SP}}\right)^{2}}{q_{0 k}^{\mathrm{SP}}} \frac{\beta_{0 l i}^{2}}{\beta_{00 k}} \\
& +\frac{1}{\gamma_{0 k}^{\mathrm{SP}}}\left(\sum_{l \in \Phi} \sum_{i=1}^{K}\left(q_{l i}^{\mathrm{SP}}+\rho_{l i}^{\mathrm{SP}}\right) \beta_{0 l i}+\sigma^{2}\right) .
\end{aligned}
$$

When perfect pilot subtraction is assumed, the capacity lower bound is 
given by (55) with the effective SINR defined as

$$
\left.\begin{array}{rl}
\operatorname{SINR}_{0 k}^{\mathrm{UL}-\mathrm{SP}-\mathrm{UB}} & =M \rho_{0 k}^{\mathrm{SP}} \beta_{00 k} / \underbrace{\frac{M}{\tau_{u l}} \sum_{l \in \Phi \backslash 0} \sum_{i=1}^{K} \frac{\rho_{l i}^{\mathrm{SP}} q_{l i}^{\mathrm{SP}}}{q_{0 k}^{\mathrm{SP}}} \frac{\beta_{0 l i}^{2}}{\beta_{00 k}}}_{\begin{array}{c}
\text { Coherent interference caused } \\
\text { by pilot contamination }
\end{array}}+\underbrace{\frac{M}{\tau_{u l}} \sum_{l \in \Phi} \sum_{i=1}^{K} \frac{\left(\rho_{l i}^{\mathrm{SP}}\right)^{2}}{q_{0 k}^{\mathrm{SP}}} \frac{\beta_{0 l i}^{2}}{\beta_{00 k}}}_{\begin{array}{c}
\text { Coherent interference } \\
\text { caused by data symbols }
\end{array}} \\
& +\underbrace{\frac{1}{\tau_{u l}^{2}} \sum_{l \in \Phi} \sum_{i=1}^{K} \frac{\left(\rho_{l i}^{\mathrm{SP}}\right)^{2}}{q_{0 k}^{\mathrm{SP}}} \frac{\beta_{0 l i}^{2}}{\beta_{00 k}}+\frac{1}{\gamma_{0 k}^{\mathrm{SP}}}\left(\sum_{l \in \Phi} \sum_{i=1}^{K} \rho_{l i}^{\mathrm{SP}} \beta_{0 l i}+\sigma^{2}\right.}_{\text {Non-coherent interference plus noise }}))
\end{array}\right) .
$$

Proof. I follows from the analysis shown in Section 2.4, combined with (50), and it is given in Paper A.

In the DL, the capacity analysis is essentially the same as the one shown in Section 2.4 with the difference that the received signal is taken at $\mathrm{UE}_{0 k}$. In this case $\mathrm{BS}_{0}$ performs a precoding operation where the transmitted signal at an arbitrary sample of the coherence block is $\mathbf{A}_{0} \mathbf{x}_{0}$. Here, $\mathbf{x}_{0}=\left[x_{01}, \ldots, x_{0 K}\right]^{T}$ is a vector containing the data symbol (including the power scaling) intended to each UE in cell 0 . The matrix $\mathbf{A}_{0} \in \mathbb{C}^{M \times K}$ is called the precoding matrix and it is meant to process the data symbols in such a way that the signals containing $x_{0 k}$ add coherently at the location of $\mathrm{UE}_{0 k}$. This allows $K$ parallel data streams to be supported in each coherence block. In this thesis, maximum ratio transmission (MRT) processing is assumed, where the precoding matrix is selected as $\mathbf{A}_{0}=\hat{\mathbf{H}}_{0}^{*}$. The SE expressions in the DL with $\mathrm{RP}$, and SP are given as follows.

\section{DLSE with RP:}

Lemma 4. A lower bound on the DL capacity of $\mathrm{UE}_{0 k}$ with RP and MRT precoding is

$$
\mathrm{R}_{0 k}^{\mathrm{DL}-\mathrm{RP}}=\frac{\tau_{d l}}{\tau_{c}} \log _{2}\left(1+\mathrm{SINR}_{0 k}^{\mathrm{DL}-\mathrm{RP}}\right)
$$

where $\mathrm{SINR}_{0 k}^{\mathrm{DL}-\mathrm{RP}}$ is the effective SINR of $\mathrm{UE}_{0 k}$ given by

$$
\operatorname{SINR}_{0 k}^{\mathrm{DL}-\mathrm{RP}}=\frac{M p_{0 k}^{\mathrm{RP}} \beta_{00 k} \gamma_{0 k}^{\mathrm{RP}}}{\frac{M}{\tau_{p}^{\mathrm{RP}}} \sum_{l \in \Phi \backslash 0} \sum_{i=1}^{K} \frac{q_{0 k}^{\mathrm{RP}} p_{l i}^{\mathrm{RP}}}{q_{l i}^{\mathrm{RP}}} \frac{\beta_{l 0 k}^{2} \gamma_{l i}^{\mathrm{RP}}}{\beta_{l l i}}+\sum_{l \in \Phi} \beta_{l 0 k} \sum_{i=1}^{K} p_{l i}^{\mathrm{RP}}+\sigma^{2}} .
$$

The average transmission energy per symbol allocated by $\mathrm{BS}_{l}$ to $\mathrm{UE}_{l i}$ with $R P$ is $p_{l i}^{\mathrm{RP}}$.

\section{DLSE with SP:}


Theorem 2. A lower bound on the DL capacity of $\mathrm{UE}_{0 k}$ with SP and MRT precoding is

$$
\mathrm{R}_{0 k}^{\mathrm{DL}-\mathrm{SP}}=\frac{\tau_{d l}}{\tau_{c}} \log _{2}(1+\underbrace{\frac{M p_{0 k}^{\mathrm{SP}} \beta_{00 k} \gamma_{0 k}^{\mathrm{SP}}}{I_{\mathrm{C}-\mathrm{I}}^{\mathrm{DL}}+I_{\mathrm{NC}-\mathrm{I}}^{\mathrm{DL}}}}_{\mathrm{SINR}_{0 k}^{\mathrm{DL}-\mathrm{SP}}})
$$

where $\mathrm{SINR}_{0 k}^{\mathrm{DL}-\mathrm{SP}}$ is the effective SINR of $\mathrm{UE}_{0 k}$. The term $I_{\mathrm{C}-\mathrm{I}}^{\mathrm{DL}}$ corresponds to the coherent interference and $I_{\mathrm{NC}-\mathrm{I}}^{\mathrm{DL}-\mathrm{S}}$ is the non-coherence interference plus noise, which are given by

$$
\begin{aligned}
& I_{\mathrm{C}-\mathrm{I}}^{\mathrm{DL}-\mathrm{SP}}=\underbrace{\frac{M}{\tau_{u l}} \sum_{l \in \Phi \backslash 0} \sum_{i=1}^{K} \frac{q_{0 k}^{\mathrm{SP}} p_{l i}^{\mathrm{SP}}}{q_{l i}^{\mathrm{SP}}} \frac{\beta_{l 0 k}^{2} \gamma_{l i}^{\mathrm{SP}}}{\beta_{l l i}}}_{\text {Interference caused by pilot contamination }}+\underbrace{\frac{M}{\tau_{u l}} \sum_{l \in \Phi} \sum_{i=1}^{K} \frac{\rho_{0 k}^{\mathrm{SP}} p_{l i}^{\mathrm{SP}}}{q_{l i}^{\mathrm{SP}}} \frac{\beta_{l 0 k}^{2} \gamma_{l i}^{\mathrm{SP}}}{\beta_{l l i}}}_{\text {Interference caused by data symbols }} \\
& I_{\mathrm{NC}-\mathrm{I}}^{\mathrm{DL}-\mathrm{SP}}=\sum_{l \in \Phi} \beta_{l 0 k} \sum_{i=1}^{K} p_{l i}^{\mathrm{SP}}+\sigma^{2} .
\end{aligned}
$$

The average transmission energy per symbol allocated by $\mathrm{BS}_{l}$ to $\mathrm{UE}_{l i}$ with $S P$ is $p_{l i}^{\mathrm{SP}}$.

Proof. It can be found in Paper A.

For the numerical results, a multicell MU-MIMO random network deployment is considered. The BSs are dropped in a square area following a homogeneous Poisson point process (PPP) with a density of 100 [BSs $/ \mathrm{km}^{2}$ ] where the area is properly scaled to simulate an average number of $30 \mathrm{BSs}$. The wraparound technique is used to avoid cell edge effects. $K$ UEs per cell are distributed uniformly within the Voronoi region around each BS. The LSF is modeled as $\beta_{l l k}=\omega^{-1} d_{l l k}^{-\alpha}$ where $d_{l l k}[\mathrm{~m}]$ is the distance between $\mathrm{BS}_{l}$ and $\mathrm{UE}_{l k}$ (with minimum distance $d_{\min }=10[\mathrm{~m}]$ ), $\alpha$ is the pathloss exponent and $\omega$ is the pathloss at a reference distance of $1[\mathrm{~m}]$. The selection of simulation parameters is depicted in Table 1.

In the UL, the transmission power of UEs needs to be controlled in order to avoid that signals from UEs close to the BSs overwhelm signals from UEs further away. This is particularly important in Massive MIMO where lowresolution ADCs are expected to be used [50,51]. Thus, statistical channel inversion power control is assumed where

$$
q_{l i}^{\mathrm{RP}}=\rho_{l i}^{\mathrm{RP}}=\frac{\varrho_{u l}}{\beta_{l l i}}, \quad q_{l i}^{\mathrm{SP}}=\frac{\Delta \varrho_{u l}}{\beta_{l l i}}, \quad \rho_{l i}^{\mathrm{SP}}=\frac{(1-\Delta) \varrho_{u l}}{\beta_{l l i}},
$$




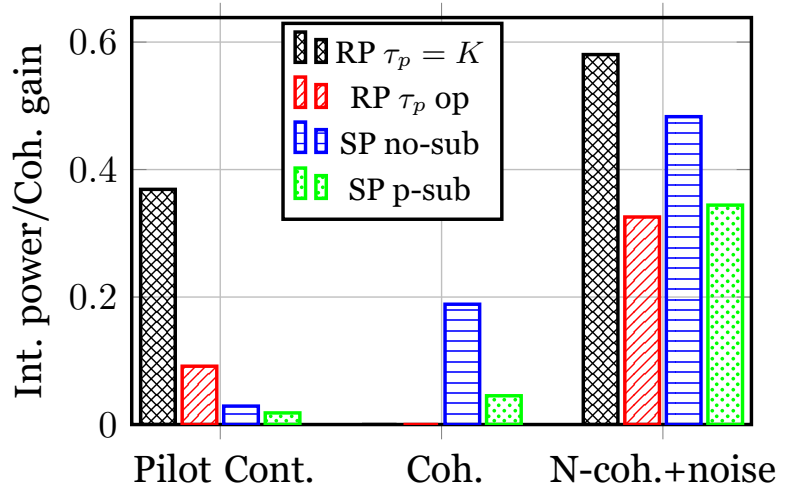

Figure 8: Interference sources in the UL for $M=100, K=10$, $\frac{\varrho_{u l}}{\sigma^{2}}=0[\mathrm{~dB}]$, and $\tau_{c}=\tau_{u l}=200$.

and $\Delta \in[0,1]$ is the proportion between the power allocated to pilot and data symbols with SP. In all results with SP, $\Delta$ is optimized to maximize the UL SE. Notice that the power control definition in (64) assumes that the total average energy per symbol with both RP and SP is the same, that is, $q_{l i}^{\mathrm{RP}}=\rho_{l i}^{\mathrm{RP}}=q_{l i}^{\mathrm{SP}}+\rho_{l i}^{\mathrm{SP}}$.

To ease readability, a summary of the abbreviations used for the legends in Figures 8-10 is presented below:

- RP with pilot length equal to the number of UEs per cell, i.e., $\tau_{p}=K$ is denoted as " $\mathrm{RP} \tau_{p}=K$ ";

- RP with optimal pilot length to maximize the SE is denoted as "RP $\tau_{p}$ op";

- SP with no pilot subtraction is denoted as "SP no-sub";

- SP with estimated pilot subtraction is denoted as "SP e-sub";

- SP with perfect pilot subtraction is denoted as "SP p-sub";

As it was done in Section 2.5, first all the figures are introduced, and then the main insights are explained summarizing the analytical and numerical results. For the UL, Figure 8 depicts the received power from the main sources of interference normalized by the coherent gain, and Figure 9 shows the SE and EE versus the number of BS antennas. To analyze the DL, both UL and DL need to be considered since the UL estimates are used for 


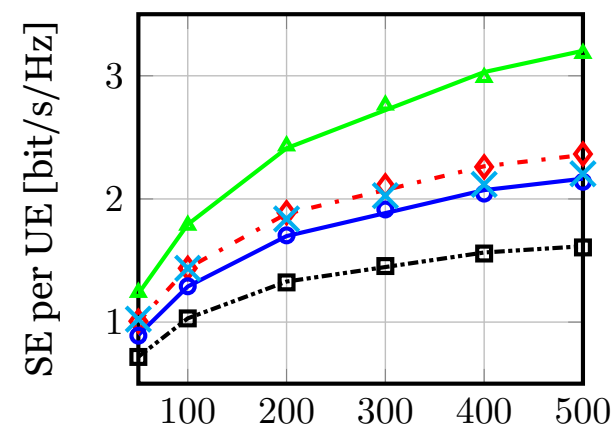

Number of BS antennas $M$

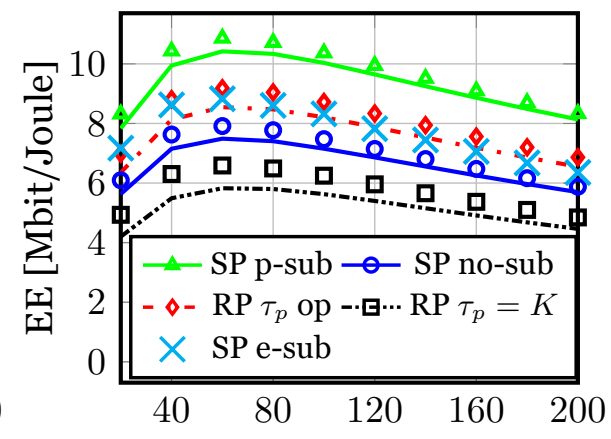

Number of BS antennas $M$

(a) SE per UE for $\frac{\underline{\varrho}_{u l}}{\sigma^{2}}=-6[\mathrm{~dB}]$, $\tau_{c}=200$ and $K=10$.

(b) EE for $\frac{\varrho_{u l}}{\sigma^{2}}=-6[\mathrm{~dB}], \tau_{c}=200$ and $K=10$.

Figure 9: SE and EE versus $M$. The markers correspond to MC simulations over the SSF. The lines in Figure 9a correspond to the closed-form expressions in Lemma 3 and Theorem 1, whereas the lines in Figure 9b correspond to the closed-form lower bounds on the SE with respect to the UE locations (see Theorem 2 in Paper A ).

DL precoding. Thus, Figure 10a depicts the DL SE per cell versus the number of BS antennas when the UL is optimized to maximize the UL rates, ${ }^{2}$ for two proportions of UL and DL symbols (i.e., values of $\tau_{u l}$ and $\tau_{d l}$ when $\tau_{c}$ is fixed). In Figure 10b the Pareto boundary ${ }^{3}$ of UL and DL sum SE over the whole network is presented for two coherence block sizes $\left(\tau_{c}=\{25,100\}\right)$. This last results is obtained based on a multiobjective optimization analysis [52] with two objectives, that is, UL and DL network SE (i.e., the sum SE over all UEs in all BSs). The optimization variables are $\tau_{u l}, \tau_{d l}$, and $\tau_{p}$ with RP, whereas, $\Delta$ with SP. The DL power allocation is done to maximize the DL sum SE per cell and an iterative algorithm is performed over all cells until convergence is achieved. In what follows, the main insights from the analytical and numerical results are explained.

\section{SP reduces pilot contamination at the expense of adding extra co- herent interference.}

Since the pilots are reused less often in space, there are less UEs that share the same pilots and they are located further apart which reduces the coherent interference caused by pilot contamination. This can be

\footnotetext{
${ }^{2}$ The optimization is done with respect to $\tau_{p}$ with RP and $\Delta$ with SP.

${ }^{3}$ The Pareto boundary corresponds to the set of attainable objectives for which no objective can be further improved without deteriorating another objective.
} 


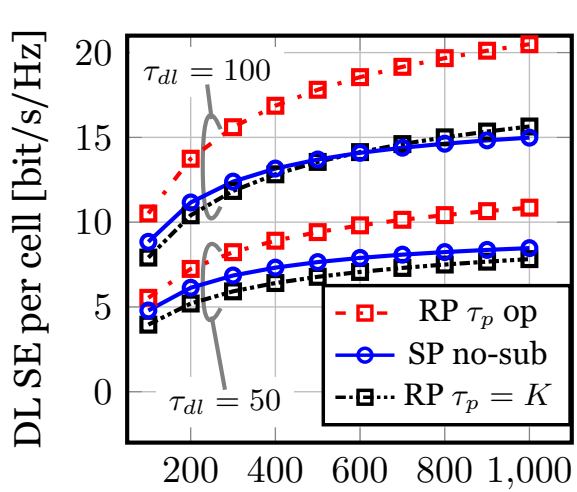

Number of BS antennas $M$

(a) DL SE per cell versus $M$ for $K=10, \tau_{c}=200$ and $\frac{\varrho_{u l}}{\sigma^{2}}=-6$ $[\mathrm{dB}]$.

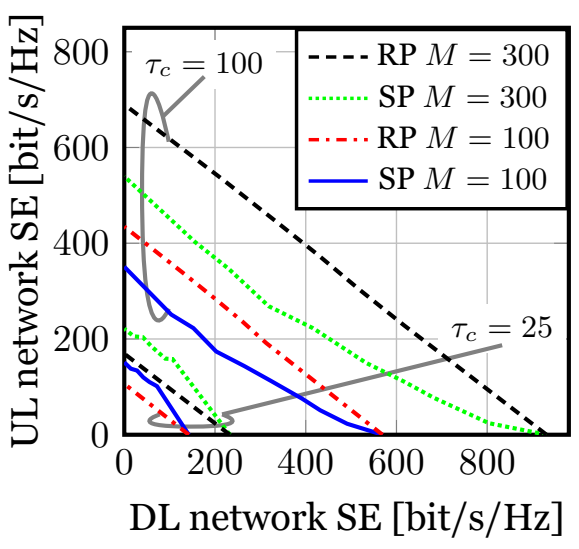

(b) Pareto boundary of UL and DL total sum network SE for $K=20$ and $\frac{\varrho_{u l}}{\sigma^{2}}=-6[\mathrm{~dB}]$.

Figure 10: DL SE per cell versus $M$, and Pareto boundary of UL and DL network SE. The DL power allocation is max-sum rate per cell. The maximum DL power is set so that the lowest cell edge SNR is equal to $\frac{\underline{\varrho}_{u l}}{\sigma^{2}}$.

seen explicitly when comparing the first term in the denominator of (54) with RP and the first term in (56) with SP, where the pilot contamination effect is reduced by a factor of $\tau_{u l} / \tau_{p}$. In turn, coherent and non-coherent interference from data symbols is added with SP as shown in the terms (56), (57), and the denominator of (58). These effects are illustrated in Figure 8 where it can be seen that the reduction in pilot contamination with SP is followed by extra interference.

\section{Correlation between channel estimates and data limits the gains with SP resulting in comparable performance with RP.}

Due to the correlation between channel estimates and UL data symbols, additional coherent and non-coherent interference is introduced with SP. In turn, this effect reduces the SE and EE gains that SP offers which can be seen in Figures 9-10. The performance when both systems, RP and SP, are optimized is comparable, one cannot say that one is clearly better than another. This is shown in the UL for the SE in Figure 9a, and the EE in Figure 9b. In the case when both UL and DL and jointly optimized the DL SE with both SP and RP are also comparable as shown in Figure 10a. Furthermore, in cases when the coherence block size is significantly larger (in the order of magnitude 
sense) than the number of multiplexed UEs, Figure 10b shows that the Pareto boundary with SP is contained within the boundary with $\mathrm{RP}$. The opposite occurs in extreme cases where $\tau_{c} \approx K$.

Thus, there is little incentive in using the alternative SP design to improve SE or EE unless the size of the coherence block is very limited and many UEs need to be multiplexed. However, in this case it may be more suitable to multiplex UEs in time or frequency, this needs to be further investigated. It is worth mentioning that, since there is an important performance gap between SP with estimated and perfect pilot subtraction, more involved signal processing like iterative decoding algorithms could provide further gains with SP.

\subsection{Hardware Impairments Optimization}

Due to the large number of BS antennas deployed in Massive MIMO, the quality of the RF chains needs to be relaxed to limit the cost. Thus, the design of the hardware needs to balance high performance with low complexity. In this section, a single cell MU-MIMO system is considered and two main research questions are investigated:

Question 1) analyzed in Paper C. What is the optimal tradeoff between the number of BS antennas and the level of hardware impairments considering SE and power consumption?

Question 2) analyzed in Paper D. Given a total ADC bit budget for the BS antenna array, what is the optimal allocation of ADC bit resolution across the antenna array in terms of maximal SE and minimum power consumption? does the alternative design with mixed ADCs offer higher SE?

In this thesis, a parametrized model is adopted to encompass the effects of non-coherent distortion caused by the hardware impairments. For the first question, the effect of the hardware impairments at the BS is modeled by a reduction of the received signal energy of $1-\epsilon^{2}$ which is turned into distortion defined as e $\in \mathbb{C}^{M \times 1}$. This assumption is made to have energy conservation after the signal is affected by hardware impairments. The parameter $\epsilon \in[0,1]$ refers to the level of hardware impairments such that $\epsilon=0$ indicates perfect hardware and as $\epsilon$ increases the hardware quality decreases. The received signal at the $\mathrm{BS}$ is

$$
\mathbf{y}=\sqrt{1-\epsilon^{2}}\left(\sum_{i=1}^{K} \mathbf{h}_{i} x_{i}+\mathbf{n}\right)+\mathbf{e}
$$


where $\mathrm{UE}_{i}$ transmits the signal $x_{i}$ and the noise is $\mathbf{n} \sim \mathcal{C N}\left(\mathbf{0}, \mathbf{I}_{M} \sigma^{2}\right)$ with average energy per symbol given by $\sigma^{2}$. The distortion is defined as

$$
\mathbf{e}=\sqrt{\epsilon^{2}\left(\sum_{i=1}^{K} \mathbb{E}\left\{\left|x_{i}\right|^{2}\right\} \mathbf{D}_{\left|\mathbf{h}_{i}\right|^{2}}+\mathbf{I}_{M} \sigma^{2}\right) \mathbf{r}}
$$

where $\mathbf{D}_{\left|\mathbf{h}_{i}\right|^{2}}=\operatorname{diag}\left(\left|\left[\mathbf{h}_{i}\right]_{1}\right|^{2}, \ldots,\left|\left[\mathbf{h}_{i}\right]_{M}\right|^{2}\right) \forall i \in\{1, \ldots, K\}$ and the randomness of the distortion is modeled by $\mathbf{r} \sim \mathcal{C N}\left(\mathbf{0}, \mathbf{I}_{M}\right)$. The model in (65) is based on Bussgang's Theorem [53], where the autocorrelation of the signal taken at the output of a nonlinear memoryless function can be expressed in terms of the autocorrelation of the input signal when the latter is assumed to be Gaussian distributed. Furthermore, a worst case Gaussian assumption is made for the randomness of the distortion (see $\mathbf{r}$ in (66)) to have a lower bound on the capacity.

For the second question, the level of hardware impairments is redefined as $\varepsilon^{2}=\epsilon^{2} /\left(1-\epsilon^{2}\right)$ to have a more compact result. The same model is used with the difference that each antenna element has an individual level of hardware impairments that is enclosed by the matrix $\mathbf{D}_{\varepsilon}=\operatorname{diag}\left(\varepsilon_{1}^{2}, \ldots, \varepsilon_{M}^{2}\right)$. The received signal at the $\mathrm{BS}$ is

$$
\mathbf{y}=\sum_{i=1}^{K} \mathbf{h}_{i} x_{i}+\mathbf{n}+\mathbf{e}
$$

and the distortion noise is defined as

$$
\mathbf{e}=\mathbf{D}_{\varepsilon}^{\frac{1}{2}}\left(\sum_{i=1}^{K} \mathbb{E}\left\{\left|x_{i}\right|^{2}\right\} \mathbf{D}_{\left|\mathbf{h}_{i}\right|^{2}}+\sigma^{2} \mathbf{I}_{M}\right)^{\frac{1}{2}} \mathbf{r} .
$$

This parametrized model is commonly used for evaluating the overall system performance of the network under the effect of residual hardware impairments $[26,54]$. Note that in this model, the distortion noise depends on the instantaneous realizations of the channel which gives good insights into the effects introduced by imperfect CSI with added distortion. However, this is an approximated model and it should be used with caution. The aim of this analysis is to find insights into the design of the hardware impairments level based on an achievable rates analysis with uncorrelated channels, and in this case, the effect of correlation between the distorted signals from different BS antennas is neglected. In the case when the ADCs are the main cause of the hardware distortions, the parametrized model gives a good approximation of the $\mathrm{SE}$ for $\mathrm{ADC}$ bit resolutions above 3 bits. This is validated 
through numerical results and will be further explained in later analysis. It should be noted that the parametrized model does not constitute an accurate representation of the physical properties of the hardware and it should not be used with this intend. For example, this model cannot be used to describe the nonlinear effects associated with out-of-band interference.

In what follows the main analytical and numerical results needed to answer the first research question are explained along with the main insights and the answer to the question. Considering the system model in (65) and (66), a lower bound on the sum capacity based on the analysis in Section 2.4, assuming LMMSE channel estimation and MRC detection is given by ${ }^{4}$

$$
\begin{aligned}
\mathrm{R}_{\text {SUM }}(\epsilon, M)=\left(1-\frac{\tau_{p}}{\tau_{u l}}\right) \sum_{k=1}^{K} \log _{2}\left(1+\operatorname{SINR}_{k}\right), \\
\operatorname{SINR}_{k}=\left(1-\epsilon^{2}\right)^{2} M /\left(\epsilon^{2}\left(1-\epsilon^{2}\right)+\frac{2 \epsilon^{2}\left(1-\epsilon^{2}\right)\left(\frac{\epsilon^{2}}{\tau_{p}} \sum_{i=1}^{K} \frac{\beta_{i}^{2} \rho_{i}^{2}}{\beta_{k}^{2} \rho_{k}^{2}}+2\left(1-\epsilon^{2}\right)\right)}{\tau_{p}\left(\left(1-\epsilon^{2}\right)+\frac{\epsilon^{2}}{\tau_{p}} \sum_{i=1}^{K} \frac{\beta_{i} \rho_{i}}{\beta_{k} \rho_{k}}+\frac{\sigma^{2}}{\tau_{p} \beta_{k} \rho_{k}}\right)^{2}}\right. \\
+\underbrace{\left(\left(1-\epsilon^{2}\right)+\frac{\epsilon^{2}}{\tau_{p}} \sum_{i=1}^{K} \frac{\beta_{i} \rho_{i}}{\beta_{k} \rho_{k}}+\frac{\sigma^{2}}{\tau_{p} \beta_{k} \rho_{k}}\right)}_{\text {Loss in the coherent gain from imperfect CSI distortion }} \underbrace{\left(\sum_{i=1}^{K} \frac{\beta_{i} \rho_{i}}{\beta_{k} \rho_{k}}+\frac{\sigma^{2}}{\rho_{k} \beta_{k}}\right)}_{\text {Non-coherent interference }}),
\end{aligned}
$$

where the transmission energy per symbol of pilot and data from $\mathrm{UE}_{i}$ is $\rho_{i}$. It is worth mentioning that, since the distortion depends on the exact realizations of the channel (see (66)) the observations used for channel estimation are non-Gaussian, and therefore, LMMSE channel estimation is used. To facilitate later analysis, $\mathrm{R}_{\mathrm{SUM}}(\epsilon, M)$, is defined as a function of $\epsilon$ and $M$. This work assumes that the ADCs are the main source of power consumption that relates to the level of hardware impairments and the following relationship is established, $\epsilon=\zeta 2^{-b}$, where $1<\zeta<2$ is a constant related to the saturation level of the $\mathrm{ADC}[27,55]$. The power consumption is modeled as in Section 2.5 where $\mathrm{P}_{\mathrm{CD}}=0$ in order to focus on the power consumed by the circuitry that is related to the hardware impairments. Then the power consumption is also defined as a function of $\epsilon$ and $M$ given by (see (30), (32), and (33))

$$
\mathrm{P}(\epsilon, M)=\mathrm{C}_{0}+\mathrm{D}_{0} M+\mathrm{D}_{1} 2 M \zeta^{\varphi} \epsilon^{-\varphi}+\frac{B_{\mathrm{W}}}{L} 2 M K \log _{2}\left(\frac{\zeta}{\epsilon}\right)
$$

\footnotetext{
${ }^{4}$ Notice that this result assumes the same power allocation for pilot and data symbols.
} 


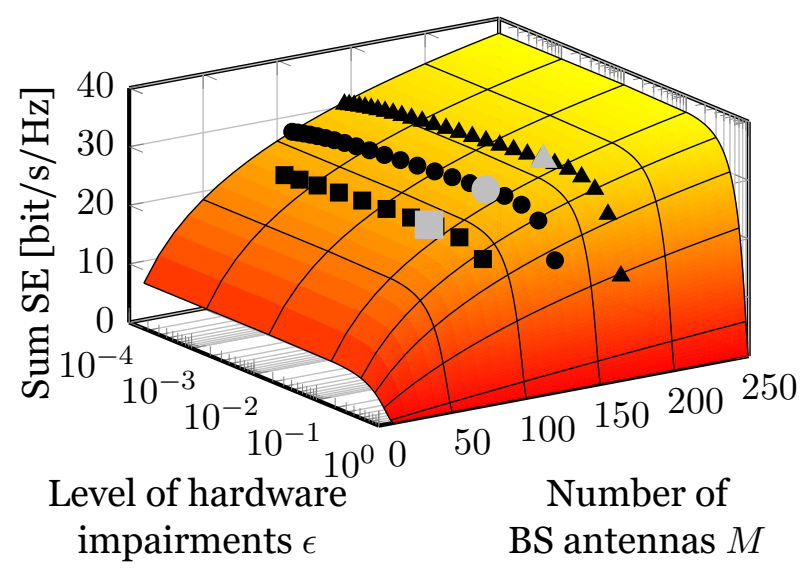

Figure 11: Sum SE versus $M$ and $\epsilon$ for $K=10$, and $\frac{\varrho_{u l}}{\sigma^{2}}=-5$ $[\mathrm{dB}]$. The dark square, circle and triangle points represent the curves where $\mathrm{P}(\epsilon, M)=\gamma$ for $\gamma=\{22,26,30\}$ [W] respectively, and the clear points are the corresponding maximum SE.

where $\mathrm{C}_{0}$ represents all terms that are independent of $\epsilon$ and $M$. To find the optimal tradeoff between $\epsilon$ and $M$ in terms of $\mathrm{R}_{\mathrm{SUM}}(\epsilon, M)$ and $\mathrm{P}(\epsilon, M)$, the following optimization problem is formulated

$$
\begin{array}{cc}
\underset{\epsilon \in[0,1], M \in \mathbb{Z}_{+}}{\operatorname{maximize}} & \mathrm{R}_{\mathrm{SUM}}(\epsilon, M), \\
\text { subject to } & \mathrm{P}(\epsilon, M) \leq \gamma
\end{array}
$$

where $\gamma[\mathrm{W}]$ is the power constraint at the BS. The optimal tradeoff that solves the optimization in (72) is given in the following lemma.

Lemma 5. Assuming that the optimization problem (72) is feasible, then the maximum $\mathrm{R}_{\mathrm{Sum}}$ is found when $\mathrm{P}(\epsilon, M)=\gamma$, thus the optimal values of $\epsilon$ and $M$ are related as

$$
M=\frac{\gamma-\mathrm{C}_{0}}{\mathrm{D}_{0}+2 \mathrm{D}_{1} \zeta^{\varphi} \epsilon^{-\varphi}+\frac{B_{\mathrm{W}}}{L} 2 K \log _{2}\left(\frac{\zeta}{\epsilon}\right)} .
$$

Proof. It follows from the monotonicity of the functions $\mathrm{R}_{\mathrm{SUM}}(\epsilon, M)$ and $\mathrm{P}(\epsilon, M)$. A more detailed proof can be found in Paper C.

Numerical results are presented to illustrate the optimal tradeoff between $\epsilon$ and $M$, along with the SE and EE performance. Statistical channel inversion power control is used for pilot and data symbols so that $\rho_{k}=\varrho_{u l} / \beta_{k}$ 


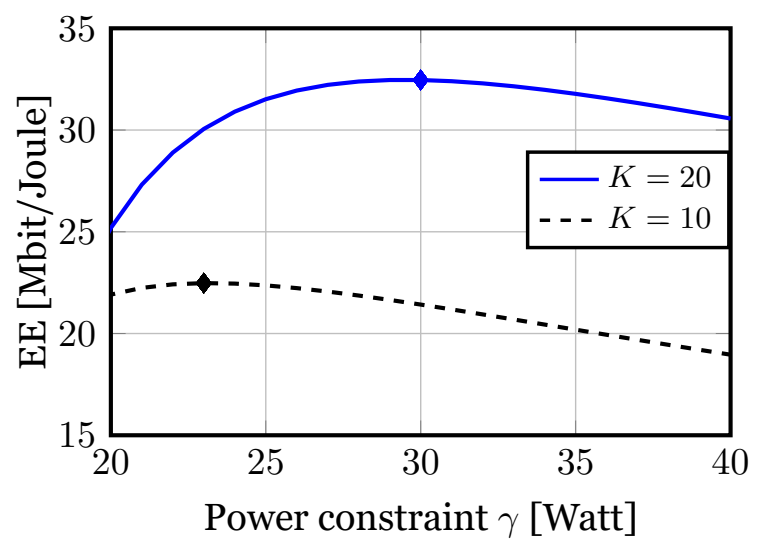

Figure 12: EE versus $\gamma$ for $K=\{10,20\}$, and $\frac{\varrho_{u l}}{\sigma^{2}}=-5$ [dB]. The marks represent the maximum EEs located at $\gamma^{*}=\{26,30\}$ [W], which correspond to $M^{*}=\{126,164\}$ and $\epsilon^{*}=\{0.056,0.0795\}$ respectively.

where $\beta_{k}$ is the LSF coefficient defined as in Section 2.5. The main parameters used in this study are depicted in Table 1. Figure 11 illustrates the sum SE versus the level of hardware impairments and the number of BS antennas. The optimal condition in (73) is depicted by the dark marks for $\gamma=\{22,23,26\}[\mathrm{W}]$ and the corresponding maximum sum SE is found at $M=\{87,126,164\}$ and $\epsilon=\{0.055,0.056,0.056\}$ which corresponds to an $\mathrm{ADC}$ resolution of $b \approx 4$ or 5 bits. By varying the power constraint $\gamma$, maximal EE can be achieved which is shown in Figure 12.

Answer 1) The optimal tradeoff between number of BS antennas and the level of hardware impairments is given by (73) as a result of solving the optimization problem in (72). Based on numerical results, the optimal solution that maximizes SE and EE lies in a Massive MIMO setup with hundreds of antennas, and $b \approx 4$ or 5 bits of $\mathrm{ADC}$ resolution.

Similarly as before, to answer the second question, the main analytical and numerical results are explained along with the main insights, and then, the answer to the second question is given. In this case, The analysis considers the system model in (67) and (68). A lower bound on the capacity based on the analysis in Section 2.4, assuming LMMSE channel estimation and MRC detection is given by

$$
\mathbf{R}_{k}=\left(1-\frac{\tau_{p}}{\tau_{u l}}\right) \log _{2}\left(1+\operatorname{SINR}_{k}\right)
$$




$$
\begin{aligned}
\operatorname{SINR}_{k}=\operatorname{tr}\left(\mathbf{D}_{z}^{-1}\right)^{2} \rho_{k} \beta_{k} / & \operatorname{tr}\left(\mathbf{D}_{\varepsilon} \mathbf{D}_{z}^{-2}\right) \rho_{k} \beta_{k} \\
& +\frac{1}{K} \operatorname{tr}\left(\mathbf{D}_{\varepsilon}\left(\mathbf{I}_{M}+\mathbf{D}_{\varepsilon}\right) \mathbf{D}_{z}^{-2}\right) \sum_{i=1}^{K} \frac{q_{i} \beta_{i}}{q_{k} \beta_{k}} \rho_{i} \beta_{i} \\
& \left.+\left(\sum_{i=1}^{K} \beta_{i} \rho_{i}+\sigma^{2}\right) \operatorname{tr}\left(\left(\mathbf{I}_{M}+\mathbf{D}_{\varepsilon}\right) \mathbf{D}_{z}^{-1}\right)\right), \\
\mathbf{D}_{z}=\mathbf{I}_{M}+\frac{1}{K} \mathbf{D}_{\varepsilon} \sum_{i=1}^{K} \frac{q_{i} \beta_{i}}{q_{k} \beta_{k}} & +\frac{\sigma^{2}}{K q_{k} \beta_{k}}\left(\mathbf{I}_{M}+\mathbf{D}_{\varepsilon}\right),
\end{aligned}
$$

where $\mathrm{UE}_{k}$ transmits data with an average energy per symbol of $\rho_{k}$ and pilots with an energy per symbol of $q_{k}$.

Here, the idea is to analyze the effect of having mixed ADC bit resolution across the antenna array at the BS. For this purpose, the same relationship between hardware impairments and $\mathrm{ADC}$ bit resolution as in previous analysis is used, that is, $\varepsilon_{m}=\zeta_{m} 2^{-b_{m}}$ where $b_{m}$ is the ADC bit resolution of the $m^{\text {th }} \mathrm{RF}$ chain. Majorization theory is used to obtain insights into the effect of having mixed ADCs when a total budget of ADC bits is assumed fixed, that is, $\sum_{m=1}^{M} b_{m}=\bar{b}$ where $\bar{b}$ is the total number of ADC bits. For mathematical tractability, $b_{m} \in \mathbb{R}$ is assumed to be a continuous variable, then consider the following definitions.

Definition 1. Let $\bar{b} \geq 1, \mathbf{b}=\left[b_{1}, \ldots, b_{M}\right]^{T}$ with $b_{1} \geq b_{2} \geq \cdots \geq b_{M} \geq 1$ and

$$
\mathcal{L}=\left\{\mathbf{b}: \sum_{m=1}^{M}[\mathbf{b}]_{m}=\bar{b}\right\} .
$$

Then, for $\mathbf{b}_{1}, \mathbf{b}_{2} \in \mathcal{L}$

$$
\mathbf{b}_{1} \succ \mathbf{b}_{2} \text { if } \sum_{m=1}^{l}\left[\mathbf{b}_{1}\right]_{m} \geq \sum_{m=1}^{l}\left[\mathbf{b}_{2}\right]_{m}, l \in\{1, \ldots, M-1\} .
$$

When $\mathbf{b}_{1} \succ \mathbf{b}_{2}$ it is said that $\mathbf{b}_{1}$ majorizes $\mathbf{b}_{2}$ [38]. In other words, the total amount of ADC bits $\bar{b}$ is spread less equally in $\mathbf{b}_{1}$ than in $\mathbf{b}_{2}$.

Definition 2. A function $f(\boldsymbol{b}): \mathbb{R}^{M} \rightarrow \mathbb{R}$ is said to be Schur-convex (Schurconcave) iffor any $\mathbf{b}_{1}, \mathbf{b}_{2} \in \mathcal{L}$ it holds that

$$
\mathbf{b}_{1} \succ \mathbf{b}_{2} \Rightarrow \begin{cases}f\left(\mathbf{b}_{1}\right) \geq f\left(\mathbf{b}_{2}\right) & \text { Schur-convex } \\ f\left(\mathbf{b}_{1}\right) \leq f\left(\mathbf{b}_{2}\right) & \text { Schur-concave }\end{cases}
$$


The idea is to find if the SE and power consumption preserve the ordering in Definition 1, that is, if it is more beneficial to have the ADC bits evenly spread across the antenna array or to concentrate them into a few high resolution ADCs. By assuming statistical channel inversion power control, that is $q_{k}=\varrho_{u l-p} / \beta_{k}$ and $\rho_{k}=\varrho_{u l-d} / \beta_{k}$, the effective SINR in (74) can be rewritten as a function of $\mathbf{b}$ given by

$$
\operatorname{SINR}_{k}(\mathbf{b})=\frac{\left(\sum_{m=1}^{M} f\left([\mathbf{b}]_{m}\right)\right)}{\sum_{m=1}^{M} g\left([\mathbf{b}]_{m}\right)}
$$

such that

$$
\begin{aligned}
& f(b)=\frac{1}{(1+\overline{\mathrm{B}})\left(1+\zeta^{2} 2^{-2 b}\right)}, \\
& g(b)=\frac{\zeta^{2} 2^{-2 b}\left(2+\zeta^{2} 2^{-2 b}\right)}{(1+\overline{\mathrm{B}})^{2}\left(1+\zeta^{2} 2^{-2 b}\right)^{2}}+\frac{\mathrm{B}}{(1+\overline{\mathrm{B}})}, \\
& \mathrm{B}=K+\frac{\sigma^{2}}{\varrho_{u l-d}}, \quad \overline{\mathrm{B}}=\frac{\sigma^{2}}{K \varrho_{u l-p}} .
\end{aligned}
$$

Notice that $f(b)$ is a concave function and $g(b)$ is convex for $b \geq 1.5$ (the proof is found in Paper D). Thus, it can be shown by evaluating the Schur's condition [38, Ch. 3] on (78), that the SE is a Schur-concave function which means that it is maximized when the ADC bits are spread equally across the antenna array. On the other hand, the power consumption as a function of $\boldsymbol{b}$ (based on the same model as in (71)) is

$$
\mathbf{P}(\mathbf{b})=\overline{\mathbf{C}}_{0}+\frac{2 K B_{\mathrm{W}}}{L} \sum_{m=1}^{M}[\mathbf{b}]_{m}+2 \mathbf{D}_{1} \sum_{m=1}^{M} 2^{\varphi_{m}[\mathbf{b}]_{m}}
$$

where $\overline{\mathrm{C}}_{0}$ includes all the power consumption terms that are independent of $b$. Thus, it can be shown by evaluating the Schur's condition [38, Ch. 3] that the power consumption is a Schur-convex function which is minimized when the ADC bits are spread evenly across the antenna array.

To illustrate the order preserving properties of the SE and power consumption, numerical analysis is conducted considering a single cell Massive MIMO system where the BS deploys $M$ antennas that serve $K=10$ UEs. The power parameters for pilot and data symbols are set to the same value, that is $\varrho_{u l-p}=\varrho_{u l-d}=\varrho_{u l}$. Figure 13 depicts the SE and the power consumption versus the $\mathrm{ADC}$ resolution spread parameter $c \in[0,1]$. This parameter is defined to quantify how equal the $\mathrm{ADC}$ bits are allocated across the antenna array. The extreme cases indicate: $i) c=0$, one antenna has 


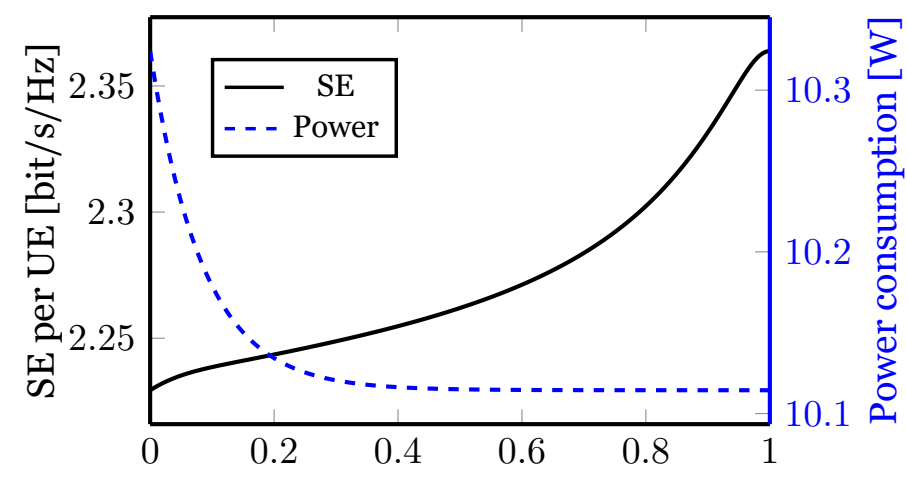

ADC resolution spread parameter $c$

Figure 13: $\mathrm{SE}$ and power consumption versus the $\mathrm{ADC}$ resolution spread parameter $c$ for $M=100, K=10, \zeta_{m}=1.6 \forall m$, $\frac{\varrho_{u l}}{\sigma^{2}}=0[\mathrm{~dB}], b^{\max }=18$.

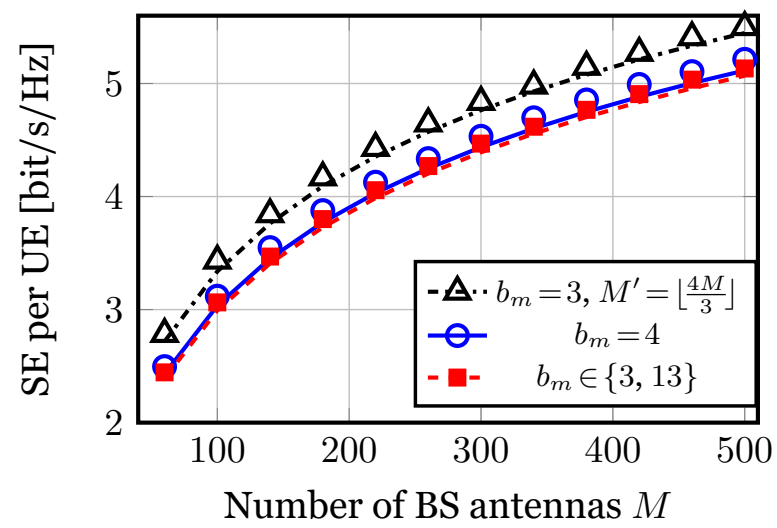

Figure 14: SE per UE versus $M$ for $\frac{\varrho_{u l}}{\sigma^{2}}=0[\mathrm{~dB}]$ and $K=10$. The markers correspond to (74) and the lines to exact quantization.

ADC resolution $b^{\max }>1$ while all the others have one bit. ii) $c=1$, all antennas have the same $\mathrm{ADC}$ resolution. It can be seen that having equal $\mathrm{ADC}$ bit resolution across the antenna array jointly maximizes $\mathrm{SE}$ and minimizes the power consumption. However, the difference in performance is rather small (in the order of magnitude sense).

Next, to validate the insights obtained from the parametrized model, a numerical analysis with exact quantization as the one shown in Section 2.5 is presented. The quantization levels are optimized as in [49] for ADC bit resolutions of up to $b_{m}=5$ bits and for $b_{m}>5$ bits with uniform levels 


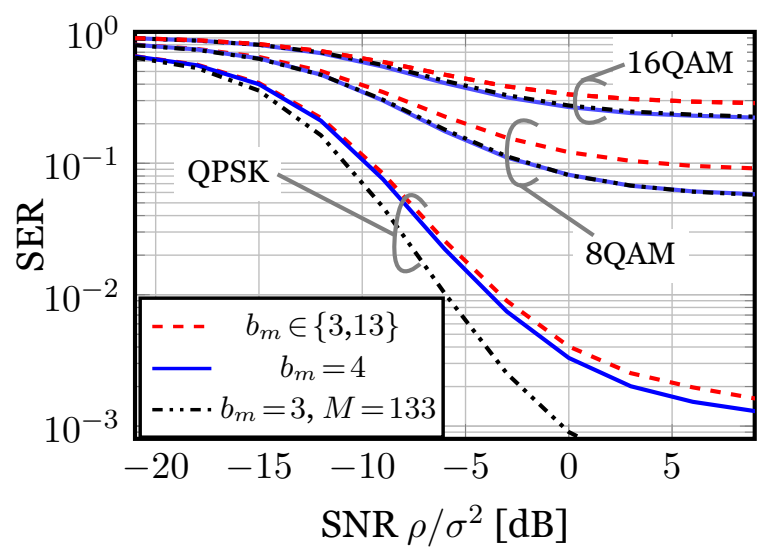

Figure 15: SER per UE versus SNR for $M=100$ and $K=10$.

optimized as in [56]. Other main parameters are given in Table 1.

To compare the performance between equal and mixed ADCs, three cases are studied: $i$ ) Mixed resolution ADCs with $M$ antennas where $10 \%$ of them have 13 bits and the rest have 3 bits. ii) Equal-resolution ADCs of 4 bits with $M$ antennas. iii) Equal-resolution ADCs of 3 bits with a larger array of $M^{\prime}=\lfloor 4 M / 3\rfloor$ antennas. Note that the sum of $\mathrm{ADC}$ bits in the whole array is almost the same for all cases, that is $\sum_{m=1}^{M} b_{m} \approx 4 M$. In Figure 14 the SE per UE is shown versus the number of $\mathrm{BS}$ antennas and it can be seen that having equal $\mathrm{ADC}$ resolutions gives higher SE even though the difference tends to be small. Furthermore, these results confirm the validity of the parametrized model used in this thesis. Figure 15 shows symbol error rate (SER) curves with exact quantization and QPSK, 8-QAM and 16-QAM symbols versus the SNR. The results show that having equal-resolution ADCs results in lower SER.

Answer 2) Given a total ADC bits budget for the BS antenna array, having equal ADC bit resolutions across the antennas maximizes SE and minimizes power consumption. Thus, the alternative design with mixed ADCs and MRC does not offer higher SE than the baseline Massive MIMO design. However, the performance loss of having mixed ADCs is rather small (in the order of magnitude sense). 
3 Alternative Massive MIMO Designs 


\section{Chapter 4}

\section{Summary, Conclusions and Future Work}

The high demand for data traffic and connected devices calls for the development of new ICT technologies. Massive MIMO has been proposed as a key enabler of next generation wireless networks for its ability to increase SE and EE. In this thesis, two alternative designs for Massive MIMO systems are studied and compared to the baseline Massive MIMO operation in terms of SE, power consumption, and EE.

The first alternative design is SP, which is based on superimposing pilot and data symbols in the UL to remove the pilot overhead and reduce the pilot contamination. To evaluate the performance with SP, novel closed-form expressions for lower bounds on the UL capacity using MRC are found. The results show that $\mathrm{SP}$ is able to reduce the pilot contamination at the expense of adding extra coherent and non-coherent interference that limits the SE gain. A comparative analysis between RP and SP in terms of the UL SE and EE indicates that, when both system are optimized, RP and SP offer comparable performance and in most cases RP with optimal pilot length is the preferred choice. However, it is worth mentioning that the SE and EE of SP with perfect pilot subtraction are higher than those with the other methods. Thus, SP may still have room for improvement and iterative decoding algorithms may help attain these gains. Furthermore, a multiobjective optimization framework is used to compare the joint UL and DL SE between RP and SP. The results show that in most cases of interest, when the number of spatial multiplexed UEs is smaller than the coherence block size, conventional RP attains higher UL and DL SE. However, the case in which the coherence block size and number of spatial multiplexed UEs are approximately the same, then SP offers higher UL and DL SE. 
The second alternative design refers to the use of mixed ADCs at the BS antenna array. The idea is to allow each RF chain to have a different ADC bit resolution and find the optimal allocation of ADC bits across the BS antennas. In this case, first a general study of the optimal tradeoff between the level of hardware impairments and number of BS antennas considering the SE and power consumption is done by using a parametrized model for the hardware impairments. Here, equal ADC bit resolution is assumed and the results show that a Massive MIMO setup with 4 or 5 bit per ADC, maximizes the SE under a power consumption constraint that, in turn, can be tuned to achieve maximal EE. Second, the case of allowing different ADC bit resolutions in each RF chain is considered under a fixed sum of ADC bits in the BS antenna array, and the same parametrize model for hardware impairments as before. Majorization theory is used to study the order preserving properties of the SE and the power consumption. The results show that maximal SE and minimum power consumption is attained when all ADC bits are equal across the antenna array. Finally, numerical results validate the parametrized model used and corroborate the insights found in theory, that is, equal ADC bit allocation offers higher SE and lower SER.

In conclusion, the baseline operation of Massive MIMO is the preferred choice when properly optimized. Possible directions for future work are the incorporation of other signal processing methods like ZF, mMMSE, maximum likelihood detection, iterative decoding algorithms, or hybrid RP and SP schemes. Moreover, using other channel models that incorporate the effect of spatial channel correlation could give interesting new insights into $\mathrm{SP}$ or mixed ADC designs. 


\section{Bibliography}

[1] Cisco, "Visual networking index: Global mobile data traffic forecast update, 2016-2021," Tech. Rep., Feb. 2017. [Online]. Available: https://www.cisco.com/c/en/us/solutions/collateral/serviceprovider/visual-networking-index-vni/mobile-white-paper-c11520862.html

[2] Ericsson, "Ericsson mobility report," Tech. Rep., Nov. 2017. [Online]. Available: https://www.ericsson.com/assets/local/mobilityreport/documents/2017/ericsson-mobility-report-november2017.pdf

[3] D. Aziz, K. Kusume, O. Queseth, and et la., "D8.4: METIS final project report," ICT-317669-METIS, Tech. Rep., Apr. 2015. [Online]. Available: https://www.metis2020.com/wpcontent/uploads/deliverables/METIS_D8.4_v1.pdf

[4] W. V. Heddeghem, S. Lambert, B. Lannoo, D. Colle, M. Pickavet, , and P. Demeester, "Trends in worldwide ICT electricity consumption from 2007 to 2012," Computer Commun., vol. 50, pp. 64-76, Feb. 2014.

[5] A. Fehske, G. Fettweis, J. Malmodin, and G.Biczok, "The global footprint of mobile communications: The ecological and economic perspective," IEEE Trans. Commun., vol. 49, no. 8, pp. 55-62, Aug. 2011.

[6] X. Zhang and J. G. Andrews, "Downlink cellular network analysis with multi-slope path loss models," IEEE Trans. Commun., vol. 63, no. 5, pp. 1881-1894, May 2015.

[7] T. L. Marzetta, E. G. Larsson, H. Yang, and H. Q. Ngo, Fundamentals of Massive MIMO. Cambridge Press, 2016.

[8] E. Björnson, J. Hoydis, and L. Sanguinetti, "Massive MIMO networks: Spectral, energy, and hardware efficiency," Foundations and Trends ${ }^{\circledR}$ in Signal Processing, vol. 11, no. 3-4, pp. 154-655, Nov. 2017. 
[9] J. Vinogradova, E. Björnson, and E. G. Larsson, "On the separability of signal and interference-plus-noise subspaces in blind pilot decontamination," in Proc. IEEE ICASSP, Mar. 2016, pp. 3421-3425.

[10] H. Q. Ngo and E. G. Larsson, "EVD-based channel estimation in multicell multiuser MIMO systems with very large antenna arrays," in Proc. IEEE ICASSP, Mar. 2012, pp. 3249-3252.

[11] R. R. Müller, L. Cottatellucci, and M. Vehkaperä, "Blind pilot decontamination," IEEE J. Sel. Topics Signal Process., vol. 8, no. 5, pp. 773786, Oct. 2014.

[12] H. Huh, G. Caire, H. C. Papadopoulos, and S. A. Ramprashad, "Achieving "massive MIMO" spectral efficiency with a not-so-large number of antennas," IEEE Trans. Wireless Commun., vol. 11, no. 9, pp. 32263239, Sep. 2012.

[13] H. Yin, D. Gesbert, M. Filippou, and Y. Liu, "A coordinated approach to channel estimation in large-scale multiple-antenna systems," IEEE J. Sel. Areas Commun., vol. 31, no. 2, pp. 264-273, Feb. 2013.

[14] E. Björnson, J. Hoydis, and L. Sanguinetti, "Massive MIMO has unlimited capacity," IEEE Trans. Wireless Commun., vol. 17, no. 1, pp. 574-590, Jan 2018.

[15] H. Yang and T. L. Marzetta, "Total energy efficiency of cellular large scale antenna system multiple access mobile networks," in Proc. IEEE OnlineGreenComm, Oct. 2013, pp. 27-32.

[16] Y. Li, Y.-H. Nam, B. L. Ng, and J. Zhang, "A non-asymptotic throughput for massive MIMO cellular uplink with pilot reuse," in Proc. IEEE GLOBECOM, Dec. 2012, pp. 4500-4504.

[17] E. Björnson, E. Larsson, and M. Debbah, "Massive MIMO for maximal spectral efficiency: How many users and pilots should be allocated?" IEEE Trans. Wireless Commun., vol. 15, no. 2, pp. 1293-1308, Feb. 2016.

[18] R. Mochaourab, E. Björnson, and M. Bengtsson, "Adaptive pilot clustering in heterogeneous massive MIMO networks," IEEE Trans. Wireless Commun., vol. 15, no. 8, pp. 5555-5568, Aug. 2016.

[19] P. Hoeher and F. Tufvesson, "Channel estimation with superimposed pilot sequence," in Proc. IEEE GLOBECOM, Dec. 1999, pp. 21622166. 
[20] G. T. Zhou, M. Viberg, and T. McKelvey, "A first-order statistical method for channel estimation," IEEE Signal Process. Lett., vol. 10, no. 3, pp. 57-60, Mar. 2003.

[21] M. Dong, L. Tong, and B. M. Sadler, "Optimal insertion of pilot symbols for transmissions over time-varying flat fading channels," IEEE Trans. Signal Process., vol. 52, no. 5, pp. 1403-1418, May 2004.

[22] A. T. Asyhari and S. ten Brink, "Orthogonal or superimposed pilots? a rate-efficient channel estimation strategy for stationary MIMO fading channels," IEEE Trans. Wireless Commun., vol. 16, no. 5, pp. 27762789, May 2017.

[23] K. Upadhya, S. A. Vorobyov, and M. Vehkaperä, "Superimposed pilots are superior for mitigating pilot contamination in massive MIMO," IEEE Trans. Signal Process., vol. 65, no. 11, pp. 2917-2932, Jun. 2017.

[24] K. Upadhya, S. A. Vorobyov, and M. Vehkaperä, "Downlink performance of superimposed pilots in massive MIMO systems," Submitted to IEEE Trans. Wireless Commun., 2017. [Online]. Available: https://arxiv.org/abs/1606.04476v2

[25] H. Zhang, S. Gao, D. Li, H. Chen, and L. Yang, "On superimposed pilot for channel estimation in multicell multiuser MIMO uplink: Large system analysis," IEEE Trans. Veh. Technol., vol. 65, no. 3, pp. 14921505, Mar. 2016.

[26] E. Björnson, J. Hoydis, M. Kountouris, and M. Debbah, "Massive MIMO systems with non-ideal hardware: Energy efficiency, estimation, and capacity limits," IEEE Trans. Inf. Theory, vol. 60, no. 11, pp. 7112-7139, Nov. 2014.

[27] E. Björnson, M. Matthaiou, and M. Debbah, "Massive MIMO with nonideal arbitrary arrays: Hardware scaling laws and circuit-aware design," IEEE Trans. Wireless Commun., vol. 14, no. 8, pp. 4353-4368, Aug. 2015.

[28] C. Desset and L. V. der Perre, "Validation of low-accuracy quantization in massive MIMO and constellation EVM analysis," in Proc. EuCNC, Jun. 2015, pp. 21-25.

[29] C. Studer and G. Durisi, "Quantized massive MU-MIMO-OFDM uplink," IEEE Trans. Commun., vol. 64, no. 6, pp. 2387-2399, Jun. 2016. 
[30] S. Jacobsson, G. Durisi, M. Coldrey, U. Gustavsson, and C. Studer, "Throughput analysis of massive MIMO uplink with low-resolution ADCs," IEEE Trans. Wireless Commun., vol. 16, no. 6, pp. 4038-4051, Jun. 2017.

[31] C. Mollén, J. Choi, E. G. Larsson, and R. W. H. Jr., "Achievable uplink rates for massive MIMO with coarse quantization," in Proc. IEEE ICASSP, Mar. 2017.

[32] C. Risi, D. Persson, and E. G. Larsson, "Massive MIMO with 1-bit ADC.” [Online]. Available: http://arxiv.org/abs/1404.7736

[33] A. Mezghani, N. Damak, and J. A. Nossek, "Circuit aware design of power-efficient short range communication systems," in Proc. IEEE ISWCS, Sep. 2010, pp. 869-873.

[34] O. Orhan, E. Erkip, and S. Rangan, "Low power analog-to-digital conversion in millimeter wave systems: Impact of resolution and bandwidth on performance," in Proc. IEEE ITA, Feb. 2015, pp. 191-198.

[35] N. Liang and W. Zhang, "Mixed-ADC massive MIMO," IEEE J. Sel. Areas Commun., vol. 34, no. 4, pp. 983-997, Apr. 2016.

[36] T. C. Zhang, C. K. Wen, S. Jin, and T. Jiang, "Mixed-ADC massive MIMO detectors: Performance analysis and design optimization," IEEE Trans. Wireless Commun., vol. 15, no. 11, pp. 7738-7752, Nov. 2016.

[37] H. Pirzadeh and L. Swindlehurst, "On the optimality of mixed-ADC massive MIMO with MRC detection," in Proc. ITG WSA, Mar. 2017.

[38] A. W. Marshall, I. Olkin, and B. C. Arnold, Inequalities : theory of majorization and its applications. Springer New York, 2011.

[39] E. Jorswieck and H. Boche, "Majorization and matrix-monotone functions in wireless communications," Foundations and Trends $\AA$ in Communications and Information Theory, vol. 3, no. 6, pp. 553-701, Jul 2007. [Online]. Available: http://dx.doi.org/10.1561/0100000026

[40] D. Tse and P. Viswanath, Fundamentals of wireless Communication. Cambridge Press, 2005.

[41] A. J. Jerri, "The shannon sampling theorem \#8212;its various extensions and applications: A tutorial review," Proceedings of the IEEE, vol. 65, no. 11, pp. 1565-1596, Nov. 1977. 
[42] X. Gao, O. Edfors, F. Rusek, and F. Tufvesson, "Massive MIMO performance evaluation based on measured propagation data," IEEE Trans. Wireless Commun., vol. 14, no. 7, pp. 3899-3911, Jul. 2015.

[43] S. M. Kay, Fundamentals of Statistical Signal Processing: Estimation Theory. Prentice Hall, 1993.

[44] H. Weingarten, Y. Steinberg, and S. S. Shamai, "The capacity region of the Gaussian multiple-input multiple-output broadcast channel," IEEE Trans. Inf. Theory, vol. 52, no. 9, pp. 3936-3964, Sep. 2006.

[45] E. Björnson, L. Sanguinetti, J. Hoydis, and M. Debbah, "Optimal design of energy-efficient multi-user MIMO systems: Is massive MIMO the answer?" IEEE Trans. Wireless Commun., vol. 14, no. 6, pp. 30593075, Jun. 2015.

[46] B. Murmann, “ADC performance survey 1997-2015," [Online]. Available: http://web.stanford.edu/ murmann/adcsurvey.html.

[47] B. Murmann, "Limits on ADC power dissipation," in Analog Circuit Design, A. H. M. Roermund and J. v. Huijsing, Eds. Springer, 2006.

[48] A. Mezghani and J. A. Nossek, "Modeling and minimization of transceiver power consumption in wireless networks," in Proc. IEEE/ITG WSA, Feb. 2011, pp. 1-8.

[49] J. Max, "Quantizing for minimum distortion," IEEE Trans. Inf. Theory, vol. 6, no. 1, pp. 7-12, Mar. 1960.

[50] S. Jacobsson, G. Durisi, M. Coldrey, U. Gustavsson, and C. Studer, "Throughput analysis of massive MIMO uplink with low-resolution ADCs," CoRR, vol. abs/1602.01139, 2016. [Online]. Available: http://arxiv.org/abs/1602.01139

[51] C. Mollén, J. Choi, E. G. Larsson, and R. W. Heath, "Uplink performance of wideband massive MIMO with one-bit ADCs," IEEE Trans. Wireless Commun., vol. 16, no. 1, pp. 87-100, Jan. 2017.

[52] E. Björnson, E. Jorswieck, M. Debbah, and B. Ottersten, "Multiobjective signal processing optimization: The way to balance conflicting metrics in 5g systems," IEEE Signal Process. Mag., vol. 31, no. 6, pp. 14-23, Nov. 2014.

[53] J. J. Bussgang, "Crosscorrelation functions of amplitude-distorted Gaussian signals," Research Laboratory of Electronics, Massachusetts Institute of Technology, Tech. Rep. 216, Mar. 1952. 
[54] U. Gustavsson, C. Sanchez-Perez, T. Eriksson, F. Athley, G. Durisi, P. Landin, K. Hausmair, C. Fager, and L. Svensson, "On the impact of hardware impairments on massive MIMO," in Proc. IEEE GLOBECOM, Dec. 2014, pp. 294-300.

[55] A. Mezghani, M. Rouatbi, and J. A. Nossek, "An iterative receiver for quantized MIMO systems," in Proc. IEEE MELECON, Mar. 2012, pp. 1049-1052.

[56] D. Hui and D. L. Neuhoff, "Asymptotic analysis of optimal fixed-rate uniform scalar quantization," IEEE Trans. Inf. Theory, vol. 47, no. 3, pp. 957-977, Mar. 2001. 


\section{Included Papers}




\section{Papers}

The papers associated with this thesis have been removed for copyright reasons. For more details about these see:

http://urn.kb.se/resolve?urn=urn:nbn:se:liu:diva-145677 


\section{Other Recently Published Theses From \\ The Division of Communication Systems Department of Electrical Engineering (ISY) Linköping University, Sweden}

Christopher Mollén, High-End Performance with Low-End Hardware: Analysis of Massive MIMO Base Station Transceivers, Linköping Studies in Science and Technology. Dissertations, No. 1896, 2017.

Trịnh Văn Chiến, Resource Allocation for Max-Min Fairness in Multi-Cell Massive MIMO, Linköping Studies in Science and Technology. Licentiate Thesis, No. 1797, 2017.

Victor Hei Cheng, Aspects of Power Allocation in Massive MIMO, Linköping Studies in Science and Technology. Licentiate Thesis, No. 1767, 2016.

Marcus Karlsson, Aspects of Massive MIMO, Linköping Studies in Science and Technology. Licentiate Thesis, No. 1764, 2016.

Christopher Mollén, On Massive MIMO Base Stations with Low-End Hardware, Linköping Studies in Science and Technology. Licentiate Thesis, No. 1756, 2016.

Antonios Pitarokoilis, Phase Noise and Wideband Transmission in Massive MIMO, Linköping Studies in Science and Technology. Dissertations, No. 1756, 2016.

Anu Kalidas M. Pillai, Signal Reconstruction Algorithms for Time-Interleaved ADCs, Linköping Studies in Science and Technology. Dissertations, No. 1672, 2015.

Ngô Quốc Hiển, Massive MIMO: Fundamentals and System Designs, Linköping Studies in Science and Technology. Dissertations, No. 1642, 2015.

Mirsad Čirkić, Efficient MIMO Detection Methods, Linköping Studies in Science and Technology. Dissertations, No. 1570, 2014.

Reza Moosavi, Improving the Efficiency of Control Signaling in Wireless Multiple Access Systems, Linköping Studies in Science and Technology. Dissertations, No. 1556, 2014.

Johannes Lindblom, The MISO Interference Channel as a Model for Non-Orthogonal Spectrum Sharing, Linköping Studies in Science and Technology. Dissertations, No. 1555, 2014.

Antonios Pitarokoilis, On the Performance of Massive MIMO Systems with Single Carrier Transmission and Phase Noise, Linköping Studies in Science and Technology. Licentiate Thesis, No. 1618, 2013.

Tumula V. K. Chaitanya, HARQ Systems: Resource Allocation, Feedback Error Protection, and Bits-to-Symbol Mappings, Linköping Studies in Science and Technology. Dissertations, No. 1555, 2013. 